\title{
Invasion and maintenance of meiotic drivers in populations of ascomycete fungi
}

\author{
Ivain Martinossi-Allibert, ${ }^{1,2}$ iD Carl Veller, ${ }^{3}$ S. Lorena Ament-Velásquez, ${ }^{1}$ Aaron A. Vogan, ${ }^{1}$ Claus Rueffler, ${ }^{4, *}$ \\ and Hanna Johannesson ${ }^{1, *}$ \\ ${ }^{1}$ Department of Organismal Biology, Uppsala University, Uppsala 75236, Sweden \\ ${ }^{2}$ E-mail: imartinossi@gmail.com \\ ${ }^{3}$ Department of Evolution and Ecology, University of California, Davis, California 95616 \\ ${ }^{4}$ Department of Ecology and Genetics, Animal Ecology, Uppsala University, Uppsala 75236, Sweden
}

Received July 8, 2020

Accepted February 25, 2021

\begin{abstract}
Meiotic drivers (MDs) are selfish genetic elements that are able to become overrepresented among the products of meiosis. This transmission advantage makes it possible for them to spread in a population even when they impose fitness costs on their host organisms. Whether an MD can invade a population, and subsequently reach fixation or coexist in a stable polymorphism, depends on the one hand on the biology of the host organism, including its life cycle, mating system, and population structure, and on the other hand on the specific fitness effects of the driving allele on the host. Here, we present a population genetic model for spore killing, a type of drive specific to fungi. We show how ploidy level, rate of selfing, and efficiency of spore killing affect the invasion probability of a driving allele and the conditions for its stable coexistence with a nondriving allele. Our model can be adapted to different fungal life cycles, and is applied here to two well-studied genera of filamentous ascomycetes known to harbor sporekilling elements, Neurospora and Podospora. We discuss our results in the light of recent empirical findings for these two systems.
\end{abstract}

KEY WORDS: Ascomycete fungi, meiosis, meiotic drive, population genetics, spore killer.

It is often assumed in genetics that the two copies of a gene in a diploid genome are represented equally among the products of meiosis - this is Mendel's first law (Lyttle 1993). However, some genetic elements called meiotic drivers (MDs) are able to distort meiosis and become overrepresented among meiotic products (Sandler and Novitski 1957; Burt and Trivers 2006). Due to this ability to distort meiosis, MDs gain a selective advantage that allows them to increase in frequency in a population even when they impose fitness costs on their host organism (Hamilton 1967; Akbari et al. 2013; Pinzone and Dyer 2013; Kyrou et al. 2018). The ensuing genetic conflict between MDs and their hosts is known to affect several evolutionary processes (Rice 2013). For example, rapid coevolution between MDs and counteracting genes, called suppressors, can accelerate speciation by creating genetic incompatibilities between recently separated populations (Frank 1991), as well as shape genetic architecture

*Shared last authors. in other important ways (Henikoff et al. 2001; Hurst and Werren 2001; Werren 2011).

MDs were discovered as early as 1928 (Sandler and Novitski 1957) and have been studied extensively since then. Early empirical observations were closely followed by theoretical work aimed at understanding the unique population genetic behavior of these selfish genetic elements (see, e.g., Lewontin and Dunn 1960; Hiraizumi 1962; Lewontin 1968, on the t-haplotype in mice). Theoretical work has focused on two key aspects of meiotic drive dynamics: under what conditions can an MD (i) invade a population and (ii) coexist at a stable equilibrium with a nondriving allele? These questions have been investigated with reference to a wide variety of species harboring MDs (e.g., Lewontin and Dunn 1960; Brand et al. 2015; Fishman and Kelly 2015; Hall and Dawe 2018), which has revealed some general patterns of MD dynamics. First, because MDs are overrepresented among successful meiotic products, theory predicts that, in the absence of counteracting forces, they should increase in frequency and reach 
fixation. However, the presence of suppressor alleles or fitness costs associated with the MD can bring the invasion process to a halt, leading ultimately to either the loss of the MD or prolonged coexistence with a nondriving allele. A typical condition for coexistence appears to be the presence of strong recessive fitness costs to the driver (or the haplotype on which it lies), allowing invasion of the MD but not fixation (e.g., Lewontin and Dunn 1960; Fishman and Kelly 2015; Holman et al. 2015). These general principles, however, are far from encompassing the complexity and diversity of MD dynamics. Indeed, although all MDs share the feature of distorting Mendelian segregation, the diversity of their modes of action as well as differences in the life cycles of their hosts make insights from one system often not applicable to others.

Here, we focus on a type of MD that has been relatively neglected by theoreticians (but see Nauta and Hoekstra 1993): spore-killing MDs in ascomycete fungi. Spore killers are found in several species of ascomycetes (Padieu and Bernet 1967; Turner and Perkins 1979; Zanders et al. 2014), and there are good reasons to expect that their dynamics differ from that of well-known MDs in plant and animal systems. The diversity of life cycles found among ascomycetes, together with the distinct mechanism of drive found in spore killers (Núñez et al. 2018), could influence the dynamics of these MDs in yet unknown ways.

In plants and animals, meiotic drive occurs during gametogenesis, and can be categorized into either female drive or male drive (reviewed in Burt and Trivers 2006; Lindholm et al. 2016). In female drive, as observed, for example, in maize (Buckler et al. 1999), monkeyflowers (Fishman and Saunders 2008), and mice (Didion et al. 2015), the MD takes advantage of the asymmetry of female meiosis by preferentially segregating to the functional egg cell (or macrospore). In contrast, in male drive, the MD kills or disables gametes that do not carry it. Well-known examples of male drive are the t-haplotype in Mus musculus (Silver 1985) and $S D$ in Drosophila melanogaster (Larracuente and Presgraves 2012).

Spore killing in fungi may appear, at first, to be similar to male drive because it also entails the killing of meiotic products that do not carry the MD (Raju 1994). However, because the dominant phase of the fungal life cycle is haploid, meiosis does not correspond to gametogenesis but instead to the production of offspring (haploid spores). Spore killing occurs when the spores produced by a single meiosis are packaged together in a sac (ascus) prior to their dispersal. Their proximity in the ascus allows spores carrying the MD to disable spores that do not carry it. Another specificity of spore killing is that it can affect all individuals in the population, instead of being limited to one sex.

The nature of the selective advantage of an MD depends on its mechanism of drive. These fall into two categories: absolute drive and relative drive (Lyttle 1991). In relative drive, the MD only increases in relative frequency by reducing the number of gametes not carrying it, while absolute drivers increase the absolute number of gametes carrying the MD (Fig. 1). The selective advantage of a purely relative driver is small during the early stages of invasion, when the driver is at low frequency (as pointed out by Nauta and Hoekstra 1993), while absolute drivers have a high initial selective advantage because they increase in absolute copy number when driving. This distinction has a large impact on the likelihood of invasion of an MD, and it is therefore important to understand to which category spore killers can belong. Before answering this question, we first examine to which categories female and male drive belong.

Female drive may be purely absolute because the MD is more likely to end up in the functional egg (or macrospore) than the alternative allele, without causing a reduction in the total number of eggs produced. In this way, the MD increases in absolute copy number (Fig. 1) with little or no fitness costs to the host. In contrast, male drive is a purely relative drive if its action results exclusively in the killing of gametes carrying the nondriving alleles: the MD increases in frequency relative to the nondriver allele, but does not increase in absolute copy number (Fig. 1).

However, male drive can enjoy an absolute advantage if the killing of sensitive meiotic products results in either the production of additional meiotic products or higher absolute fitness of surviving ones. We will refer to such benefits generally as killing advantage (Fig. 1). In male drive, killing advantage (also called "compensation") can arise when fertility does not scale linearly with the number of sperm produced (Hartl 1970): halving the sperm count may not half the offspring number, so that an MD that kills all sperm carrying the sensitive allele can end up in a greater number of offspring than expected for a neutral allele in a heterozygous parent. Killing advantage in male drive can also evolve as an adaptive response of the host organism if, for example, heterozygous carriers of the MD produce additional gametes to compensate for those killed by the MD (e.g., in the stalk-eyed fly Teleopsis dalamanni; Meade et al. 2019).

Similar considerations apply to spore killing in fungi. At first glance, spore killing appears to be a purely relative drive because spore killers directly eliminate some proportion of their host's offspring. However, spore killers might also benefit from a killing advantage, with mechanisms somewhat similar to certain forms of killing advantage in male drive. For instance, the killing of sibling spores may alleviate local competition for resources, thus increasing the viability of surviving spores. Alternatively, the parent might have a lower resource expenditure on killed spores, and thus be able to produce additional spores to compensate for the loss (Nauta and Hoekstra 1993; Lindholm et al. 2016). In both scenarios, the killing advantage provides the spore killer with a fitness benefit that makes it more akin to an absolute driver. In natural systems, it is not known whether a killing advantage 


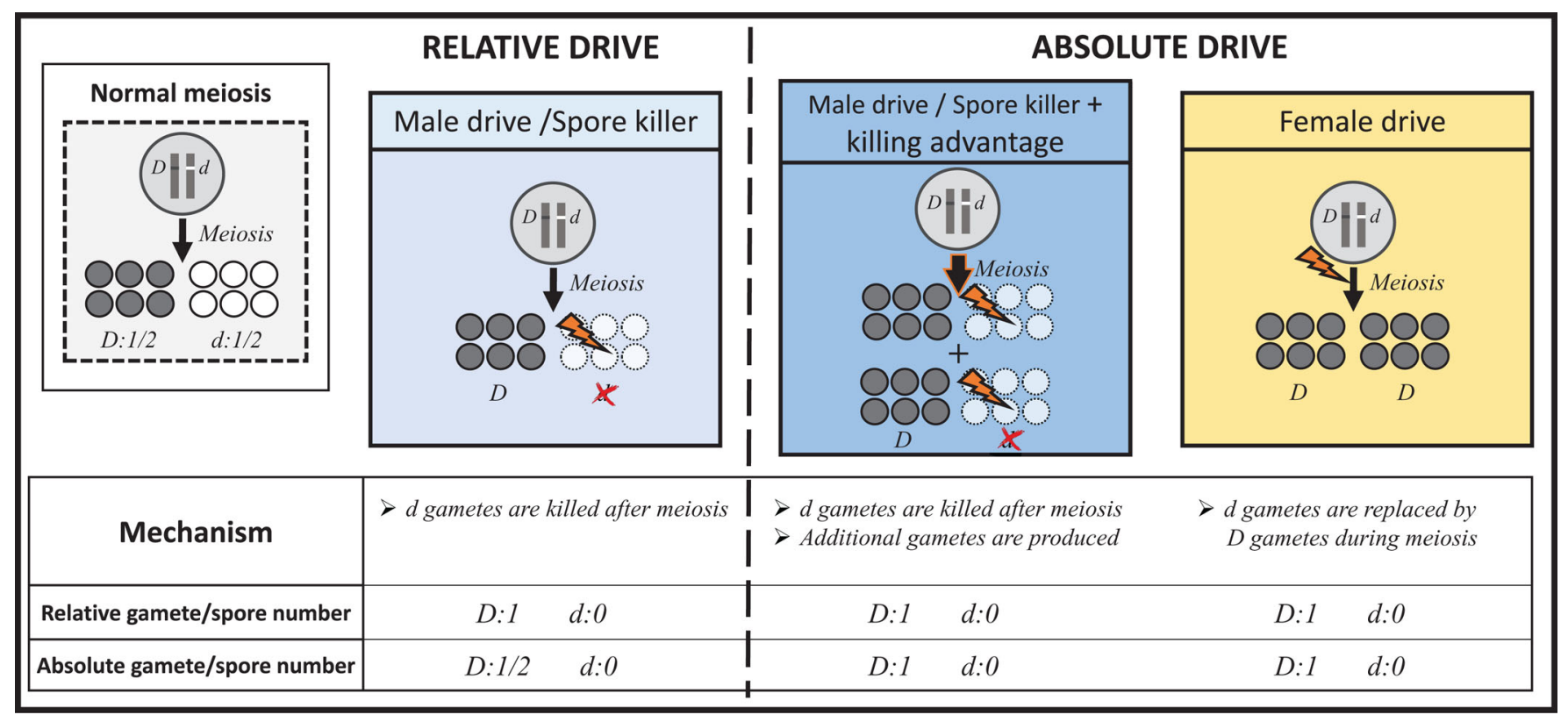

Figure 1. Relative and absolute meiotic drive. The organism is diploid and heterozygous for alleles $D$ (driving allele) and $d$ (sensitive allele) at a given locus. The figure shows how relative and absolute drive differ from normal meiosis.

generally exists for spore killers, except for the case of Podospora anserina, which has been shown to respond to spore killing with the production of additional spores (Vogan et al. 2020).

In this study, we develop a population genetic model that allows us to address the two central questions of theoretical meiotic drive studies in the relatively uncharted realm of fungal spore killers: Under what conditions can a spore-killing MD (i) invade and (ii) coexist with a sensitive allele? We investigate these questions using a single-locus model of spore killing. In doing so, we ignore-in agreement with recent empirical findings ( $\mathrm{Hu}$ et al. 2017; Nuckolls et al. 2017; Vogan et al. 2019; Svedberg et al. 2020) - the possibility of recombination between the killing and antidote functions of the spore killer, which was addressed in the only other theoretical work on spore killers we know of (Nauta and Hoekstra 1993). We cover the diversity of ascomycete life cycles by adapting our model to heterothallic Neurospora species, which are representative of typical ascomycete fungi, and $P$. anserina, which has evolved a more complex life cycle (Raju and Perkins 1994). We study the influence of fitness costs associated with the MD, killing efficiency, and killing advantage on the dynamics of the spore killer. For $P$. anserina, we also consider the impact of two additional parameters that are of interest in this case: the selfing rate and the recombination rate between the spore killer locus and the centromere. We investigate these questions by (i) performing a stability analysis of the equilibria of the recursion equation describing the dynamics of the spore killer allele and by (ii) performing stochastic simulations to assess the impact of drift on the invasion probability of a spore killer.

\section{The Model}

We study allelic dynamics at a single locus in a diploid organism with nonoverlapping generations. Two alleles segregate at the focal locus: the spore killer allele $D$ and the sensitive nonkilling allele $d$. The considered life cycles correspond to those of filamentous ascomycetes of the genera Neurospora and Podospora. Both taxa are model systems in fungal genetics and are known to harbor spore-killing elements (e.g., Silar 2013; Vogan et al. 2019; Svedberg et al. 2020). The life cycle of heterothallic Neurospora species is representative of a typical filamentous ascomycete, and the life cycle of $P$. anserina displays pseudohomothallism, an alternative reproductive strategy that has evolved many times across ascomycetes (Raju 1994). These two taxa therefore capture a wide range of the variety of ascomycete life cycles. In our analysis, we first assume that the population is sufficiently large that drift can be ignored. Under this assumption, we determine the parameter combinations that permit invasion of the spore killer allele $D$, and then ask under what further conditions invasion of $D$ results in its fixation or its stable coexistence with $d$. We then relax the assumption of an infinitely large population to explore the role of drift in the early phase of invasion of $D$.

\section{THE Neurospora MODEL}

\section{Neurospora life cycle and model parameters}

In this section, we focus on the life cycle of heterothallic $\mathrm{Neu}$ rospora species, such as $N$. sitophila and $N$. crassa, in which each individual carries only one mating type. As individuals in these 

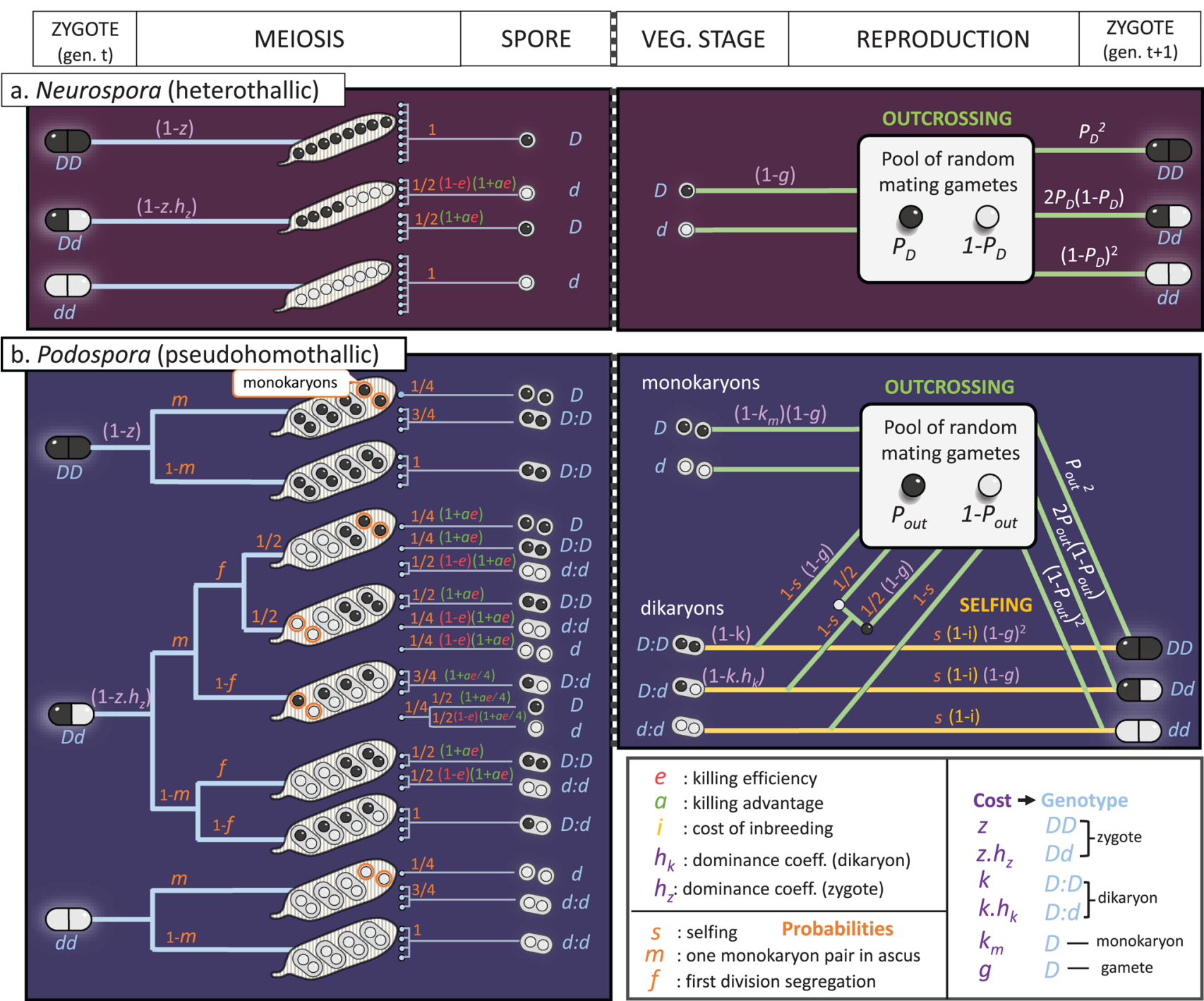

Figure 2. Life-cycle diagrams for (a) heterothallic Neurospora species and (b) P. anserina. Both life cycles are presented from zygote to zygote. The left panel shows the meiosis stage occurring shortly after zygote formation and asci representing all possible segregation patterns of $D$ and $\boldsymbol{d}$ from the three possible diploid genotypes. The right panel shows the reproductive stage with the outcrossing and selfing modes (the latter one only in Podospora) of reproduction, leading to fertilization and the formation of the diploid zygotes of the next generation. The recursion equations can be constructed by following the lines of the life-cycle diagram and multiplying each genotype frequency with the costs and probabilities that apply to it as indicated along the lines. Orange symbols represent probabilities of alternative events (e.g., selfing occurring with probability $s$ vs. outcrossing occurring with probability $1-s$ ). Purple symbols represent potential fitness costs associated with carrying the spore killer allele $D$. The cost of inbreeding at all loci (i), associated with the selfing branch of reproduction, is represented in yellow. Finally, green symbols represent a killing advantage (a) associated with spore killing and killing efficiency $(e)$ is colored in red.

species are sexually self-incompatible, we assume random mating and therefore Hardy-Weinberg proportions, although we acknowledge that inbreeding by sib-mating is a possibility in $\mathrm{Neu}$ rospora. The simple structure of the life cycle is summarized in Figure 2A. The life cycle starts with a diploid zygote that rapidly undergoes meiosis. Meiosis is followed by a mitosis that results in the formation of an eight-spore ascus. Spores disperse and grow to a haploid vegetative stage (mycelium). This vegetative stage produces haploid gametes, and the zygotes of the next generation form after fusion of gametes of opposite mating types. When an ascus originates from a zygote that is heterozygous at the spore killer locus, spore killing occurs and spores of genotype $d$ are killed with probability $e$, so that an average proportion $1-e$ of such spores survive. We refer to $e$ as the killing efficiency. When a killing event occurs, all surviving spores within the ascus (all $D$ spores and an expected fraction $1-e$ of $d$-spores) benefit from a killing advantage $a<1$. At each stage of the life cycle, carrying the $D$ allele is associated with a viability cost represented 
by parameters $z$ for the diploid stage and $g$ for the haploid stage. $D D$ diploids suffer a cost $z$, while $D d$ heterozygotes suffer a cost $z h_{z}$, where $h_{z}$ is the dominance coefficient. Figure 2A shows at what stage the different parameters apply in the life cycle of $\mathrm{Neu}$ rospora. The assumption of random mating allows us to investigate the model by following a single variable, the frequency of the $D$ allele among the pool of randomly mating gametes.

\section{Recursion equation for Neurospora}

Let $p_{D}$ be the frequency of allele $D$ in the gamete pool in the current generation, and $p_{D}^{\prime}$ its frequency in the next generation. Then

$$
p_{D}^{\prime}=\frac{p_{D}^{2} L_{D D}+p_{D}\left(1-p_{D}\right) L_{D}}{p_{D}^{2} L_{D D}+2 p_{D}\left(1-p_{D}\right)\left(\frac{L_{D}}{2}+\frac{L_{d}}{2}\right)+\left(1-p_{D}\right)^{2}},
$$

where

$$
\begin{gathered}
L_{D D}=(1-z)(1-g) \\
L_{D}=\left(1-z h_{z}\right)(1+a e)(1-g) \\
L_{d}=\left(1-z h_{z}\right)(1-e)(1+a e)
\end{gathered}
$$

are fitnesses expressed relative to the fitness of a $d$ haplotypte starting life in a $d d$ zygote (because of spore killing, selection acts on haploid genotypes, hence we consider the fitness of haplotypes). Thus, by definition $L_{d d}=1$. $L_{D D}$ represents the relative fitness of a $D$ haplotype starting life in a $D D$ zygote, $L_{D}$ the fitness of a $D$ haplotype in a $D d$ zygote, and $L_{d}$ the fitness of a $d$ haplotype in a $D d$ zygote, which, in this last case, includes the costs of being killed during the spore-killing event that follows meiosis.

\section{THE PODOSPORA MODEL}

\section{Podospora life cycle and model parameters}

Figure $2 \mathrm{~B}$ shows a schematic representation of the more complex life cycle of $P$. anserina, from which we derive a set of recursion equations describing the change in frequency of the spore killer allele $D$. A simplified biological representation of the life cycle is shown in Figure S22. Additional complexities present in $P$. anserina include a mainly dikaryotic spore packaging and vegetative stage and alternative selfing and outcrossing reproductive strategies. The life cycle starts with a zygote (left panel of Fig. 2B) that shortly after undergoes meiosis followed by one mitosis. This results in a single ascus containing eight haploid nuclei. These nuclei are packaged into pairs forming dikaryotic spores (two haploid nuclei in the same cytoplasm), or remain isolated, resulting in monokaryotic spores (haploid). In $P$. anserina, the frequency of monokaryotic spores formed in natural strains is low (van der Gaag 2005), and we assume that each ascus can contain either one pair of monokaryotic spores (with probability $m$ ) or none (with probability $1-m$ ). Heterozygous zygotes $D d$ can result in the formation of either heteroallelic $D d$ or homoallelic $D D$ and $d d$ dikaryotic spores, depending on whether the drive locus segregates reductionally (first-division segregation, or FDS) or equationally (second-division segregation, or SDS) at the first meiotic division (Rizet and Engelmann 1949). FDS at the drive locus occurs when there is either no or an even number of crossovers between the drive locus and the centromere. SDS occurs otherwise (see meiosis diagram in Fig. S22). We assume that FDS occurs at the spore killer locus with probability $f$ and results in two homoallelic spores of each genotype, while SDS occurs with probability $1-f$ and results in four heteroallelic spores (see Fig. 2B). When monokaryotic spores of genotype $d$ or dikaryotic spores of genotype $d d$ share an ascus with spores of genotype $D, D D$, or $D d$, they are killed with efficiency $e$. Dikaryotic spores of the $D d$ genotype are not affected by spore killing because the $D$ nucleus protects the entire spore from being killed (Padieu and Bernet 1967; Grognet et al. 2014). The frequencies of the different types of spores after meiosis and spore killing are denoted by $M_{D D}$, $M_{D d}, M_{d d}, M_{D}$, and $M_{d}$, respectively.

Surviving dikaryotic and monokaryotic spores germinate and form a dikaryotic or monokaryotic mycelium as the vegetative stage. We assume that dikaryons and monokaryons have the same growth rate during this stage. The vegetative stage is followed by the reproductive stage, which is represented in the right panel of Figure 2. Gametes produced by dikaryons and monokaryons enter a common pool where random mating results in diploid zygotes. In addition, dikaryons can self, which they do with probability $s$. Selfing in $P$. anserina can occur only when a dikaryon carries nuclei of the two different mating types. We assume that this is always the case because segregation of the mating-type locus is controlled by a single reciprocal crossover between the locus and the centromere, ensuring that dikaryons are heteroallelic and thus self-fertile (in nature, the probability of SDS of the mating-type locus is very close to $100 \%$ in P. anserina, Rizet and Engelmann 1949). As a consequence of this mating type constraint, heteroallelic dikaryons of genotype $D d$ can only produce $D d$ zygotes through selfing because each of the selfing haplotypes, $D$ and $d$, is associated with a particular mating type, thus preventing the formation of $D D$ or $d d$ zygotes. We note that, although this particular type of selfing maintains heterozygous genotypes during reproduction, it still results in a loss of heterozygosity because heterozygous genotypes can be broken up by FDS during meiosis resulting in homoallelic spores $(D D$ and $d d$ ). At each stage of the life cycle, our model includes the possibility of fitness costs resulting in reduced viability associated with the spore-killing allele $D$ (see Fig. 2). Costs are the same as for the Neurospora model, with the addition of a cost $k$ in the dikaryotic stage and its associated dominance coefficient $h_{k}$. 


\section{Recursion equation for a simplified Podospora model}

We first present a simplified Podospora model, which builds on the Neurospora model and only adds a dikaryotic cost parameter $k$ and the rate of FDS $f$. This simplified model does not yet include selfing or monokaryons, and therefore does not fully represent the biology of Podospora, but it is useful in allowing us to derive simple analytical results that help clarify the interpretation of the complete Podospora model introduced in the next section. Thus, assuming that the rate of selfing is zero $(s=0)$ and that monokaryons do not occur $(m=0)$, recursion (1) from the $\mathrm{Neu}$ rospora model applies to this simplified version of the Podospora model with the following modifications:

$$
\begin{gathered}
L_{D D}=(1-z)(1-k)(1-g) \\
L_{D}=\left(1-z h_{z}\right)\left(f(1+a e)(1-k)+(1-f)\left(1-k h_{k}\right)\right) \\
(1-g) \\
L_{d}=\left(1-z h_{z}\right)\left(f(1+a e)(1-e)+(1-f)\left(1-k h_{k}\right)\right) .
\end{gathered}
$$

\section{Recursion equations for the complete Podospora model} In the complete Podospora model, the occurrence of selfing implies that mating is not random, and so we need to track the frequencies of the diploid genotypes $D D, D d$, and $d d$ at the zygote stage. Each generation, some individuals from each genotype are produced through selfing and some through outcrossing. Thus, the change in frequency for the three genotypes $D D, D d$, and $d d$ is given by

$$
\begin{aligned}
p_{D D}^{\prime}= & {\left[\operatorname{selfing}_{D D}\left(p_{D D}, p_{D d}, p_{d d}\right)\right.} \\
& \left.+\operatorname{outcrossing}_{D D}\left(p_{D D}, p_{D d}, p_{d d}\right)\right] \frac{1}{\bar{W}} \\
p_{D d}^{\prime}= & {\left[\operatorname{selfing}_{D d}\left(p_{D D}, p_{D d}, p_{d d}\right)\right.} \\
& \left.+\operatorname{outcrossing}_{D d}\left(p_{D D}, p_{D d}, p_{d d}\right)\right] \overline{\bar{W}} \\
p_{d d}^{\prime}= & {\left[\operatorname{selfing}_{d d}\left(p_{D D}, p_{D d}, p_{d d}\right)\right.} \\
& \left.+\operatorname{outcrossing}_{d d}\left(p_{D D}, p_{D d}, p_{d d}\right)\right] \frac{1}{\bar{W}},
\end{aligned}
$$

where the frequencies of each genotype in the current and next generation are indicated by $p$ and $p^{\prime}$, respectively, and with genotypes indicated by subscripts, and $\bar{W}$ represents mean fitness.

The treatment of selfing and outcrossing is inspired by a plant population genetics model with self-fertilization (Holsinger et al. 1984). Beginning with the selfing part of the life cycle (right-hand panel of Fig. 2), we start from the frequencies of the three possible dikaryotic genotypes after meiosis, $M_{D D}, M_{D d}$, and $M_{d d}$. Dikaryons may pay fitness costs if they carry one or two copies of the spore killer (respectively $k h_{k}$ or $k$ ). Genotype frequencies are then adjusted by the selfing probability $s$, and all genotypes are exposed to a selfing cost $i$ due to inbreeding. Finally, because gametes are also produced during the selfing process, individuals carrying the spore killer genotype are exposed to gametic costs $g$ during selfing (as well as during outcrossing), resulting in the survival probabilities $(1-g)^{2}$ and $1-g$ for $D D$ and $D d$ dikaryons, respectively. We can now write the selfing contribution to diploid genotypes of the next generation as

$$
\begin{gathered}
\operatorname{selfing}_{D D}=w M_{D D}(1-k) s(1-i)(1-g)^{2} \\
\text { selfing }_{D d}=M_{D d}\left(1-k h_{k}\right) s(1-i)(1-g) \\
\text { selfing }_{d d}=M_{d d} s(1-i) .
\end{gathered}
$$

For the outcrossing part of the life cycle, random mating is assumed. We denote by $p_{\text {out }}$ the frequency of the spore-killing allele $D$ in the pool of randomly mating gametes. It is important to note that $p_{\text {out }}$ only represents a frequency within the outcrossing fraction of the total population, denoted by $T_{\text {out }}$. More precisely, $T_{\text {out }}$ consists of the fraction of gametes that engage in outcrossing, which are all gametes from monokaryotic individuals (potentially reduced due to fitness costs $k_{m}$ for monokaryons carrying the $D$ allele) together with the fraction $1-s$ of outcrossing gametes from dikaryons, all discounted by the appropriate reduction in viability due to costs. Genotypes produced by outcrossing follow Hardy-Weinberg proportions and are weighted by $T_{\text {out }}$ to represent valid frequencies in the total population:

$$
\operatorname{outcrossing}_{D D}=T_{\text {out }} \times p_{\text {out }}^{2}
$$

$$
\begin{gathered}
\operatorname{outcrossing}_{D d}=T_{\text {out }} \times 2 p_{\text {out }}\left(1-p_{\text {out }}\right) \\
\text { outcrossing }_{d d}=T_{\text {out }} \times\left(1-p_{\text {out }}\right)^{2},
\end{gathered}
$$

where

$$
\begin{aligned}
T_{\text {out }}= & M_{D}\left(1-k_{m}\right)(1-g)+M_{d} \\
& +(1-s)\left(M_{D D}(1-k)(1-g)+M_{D d}\left(1-k h_{k}\right)\left(1-\frac{g}{2}\right)+M_{d d}\right) \\
p_{\text {out }}= & \left(\quad M_{D}\left(1-k_{m}\right)(1-g)\right) \\
& +(1-s)\left(\left(M_{D D}(1-k)(1-g)+M_{D d}\left(1-k h_{k}\right) \frac{(1-g)}{2}\right)\right) \frac{1}{T_{\text {out }}} .
\end{aligned}
$$

The expressions for the genotype frequencies after meiosis are given by

$M_{D}=\frac{m}{4}\left(p_{D D}(1-z)+p_{D d}\left(1-z h_{z}\right)\left(\frac{f}{2}(1+a e)+(1-f) \frac{\left(1+\frac{a e}{4}\right)}{2}\right)\right)$ 
$M_{d}=\frac{m}{4}\left(p_{d d}+(1-e) p_{D d}\left(1-z h_{z}\right)\left(\frac{f}{2}(1+a e)+(1-f) \frac{\left(1+\frac{a e}{4}\right)}{2}\right)\right)$

$M_{D D}=\left(\frac{3}{4} m+(1-m)\right)\left(p_{D D}(1-z)+p_{D d}\left(1-z h_{z}\right) \frac{f}{2}(1+a e)\right)$

$$
\begin{gathered}
M_{D d}=\left(\frac{3}{4} m\left(1+\frac{a e}{4}\right)+(1-m)\right) p_{D d}\left(1-z h_{z}\right)(1-f)(8 \mathrm{~d}) \\
M_{d d}=\left(\frac{3}{4} m+(1-m)\right)\left(p_{d d}+(1-e) p_{D d}\left(1-z h_{z}\right) \frac{f}{2}(1+a e)\right)
\end{gathered}
$$

and are derived from the left-hand panel of Figure 2. Fitness costs can affect diploid zygotes, reducing the initial frequencies by the factors $1-z$ and $1-z h_{z}$ for $D D$ and $D d$ genotypes, respectively. When a pair of monokaryons are formed (with probability $m$ ), they represent $1 / 4$ of the nuclei in their ascus. Thus, monokaryotic spores represent a fraction $m / 4$ of the initial diploid frequencies, and dikaryons represent a fraction $\frac{3}{4} m+(1-m)$. Spore killing affects monokaryotic spores of the $d$ genotype and dikaryotic spores of the $d d$ genotype originating from $D d$ diploids. In these cases, killing occurs with efficiency $e$ so that a proportion $1-e$ of the sensitive spores exposed to killing survive. Importantly, monokaryotic $d$-spores are affected under both FDS and SDS, while dikaryotic spores are affected only when homoallelic $(d d)$, and therefore only under FDS (which occurs with probability $f$ ). Only under maximal killing efficiency $(e=1)$ are all sensitive spores exposed to the $D$ allele killed. In asci in which spore killing occurs, the surviving spores benefit from a killing advantage regardless of their genotype. The killing advantage can originate from additional resources made available due to spores being killed and is therefore assumed to be proportional to the number of killed spores. For this reason, the killing advantage is weighted by the killing efficiency, providing a benefit $1+$ ae to surviving spores. The killing advantage benefiting monokaryotic spores of an ascus in which the killer locus underwent SDS is special. In this case, only one nucleus per ascus can be killed in contrast to the four nuclei that are killed under FDS. When this occurs, the killing advantage is $1+\frac{a e}{4}$. Finally, $\bar{W}$ is the sum of the numerators on the right-hand side of equation (4).

\section{Expected parameter range for Podospora}

For natural population of $P$. anserina, it is unknown whether spore-killing alleles impose fitness costs and if a killing advantage exists. Therefore, we study the broadest possible range of fitness costs for each stage of the life cycle, from 0 (no costs) to 1 (fully lethal), and a wide range of killing advantages from 0 (no benefit) to 1 (all killed spores being replaced). The rate of selfing in natural populations of Podospora is also unknown. However, the propensity of Podospora species to self in laboratory conditions together with low overall levels of genetic diversity (Vogan et al. 2019) indicate that selfing may be frequent. Therefore, we study the effect of selfing rates ranging from 0 to $95 \%$. Known spore killers in Podospora undergo FDS in 30-100\% of meioses, depending on the variants (van der Gaag et al. 2000; Vogan et al. 2019). We cover this range by studying the probabilities $0.25,0.5$, and 1 of FDS. Under natural conditions, the occurrence of asci containing monokaryons varies between $0 \%$ and 6\% (Esser 1974; van der Gaag 2005). We analyze the model without monokaryons first, and then with monokaryons occurring in $5 \%$ of asci to cover the natural range, and finally in $50 \%$ of asci to make the effect of monokaryons on spore-killing dynamics more visible. Finally, the killing efficiency $e$ is believed to be high in Podospora (Vogan et al. 2019). Hence, we focus on the case with $e=1$, and only briefly explore incomplete killing $(e<1)$ to contrast its effect with that of the probability of FDS $f$ being less than 1 .

\section{METHODS}

We first analyze the deterministic recursions to characterize the parameter combinations that permit invasion with subsequent fixation of $D$, or invasion with subsequent stable polymorphism. To this end, we identify the equilibria of the recursions and determine their stability. Stability is determined based on a linear stability analysis of the one-dimensional system in the cases of $\mathrm{Neu}$ rospora and simplified $P$. anserina without selfing (Otto and Day 2011, pp. 163-172) or the two-dimensional system in the case of P. anserina with selfing (Otto and Day 2011, pp. 316-320). For the one-dimensional models we obtain analytical expressions for the values of the equilibria and their stability conditions. In the case of the complete $P$. anserina model, we cannot solve for the values of the interior equilibria symbolically and therefore conduct parameter sweeps.

To determine the invasion probability of a spore-killing allele in a finite population, we analyze a stochastic version of the model accounting for drift. In this analysis, the recursions define the sampling probabilities of a Wright-Fisher process, with sampling occurring at the stage of zygote formation. For each set of parameter values, the invasion probability is estimated as the proportion of 1000 simulation runs in which the spore killer achieves the equilibrium frequency of the deterministic system (fixation or stable polymorphism). A stochastic simulation is considered to have reached an internal equilibrium if allele frequencies fluctuate around the same value for at least 1000 generations. The invasion probability is taken to be zero whenever the deterministic model does not allow for invasion. 


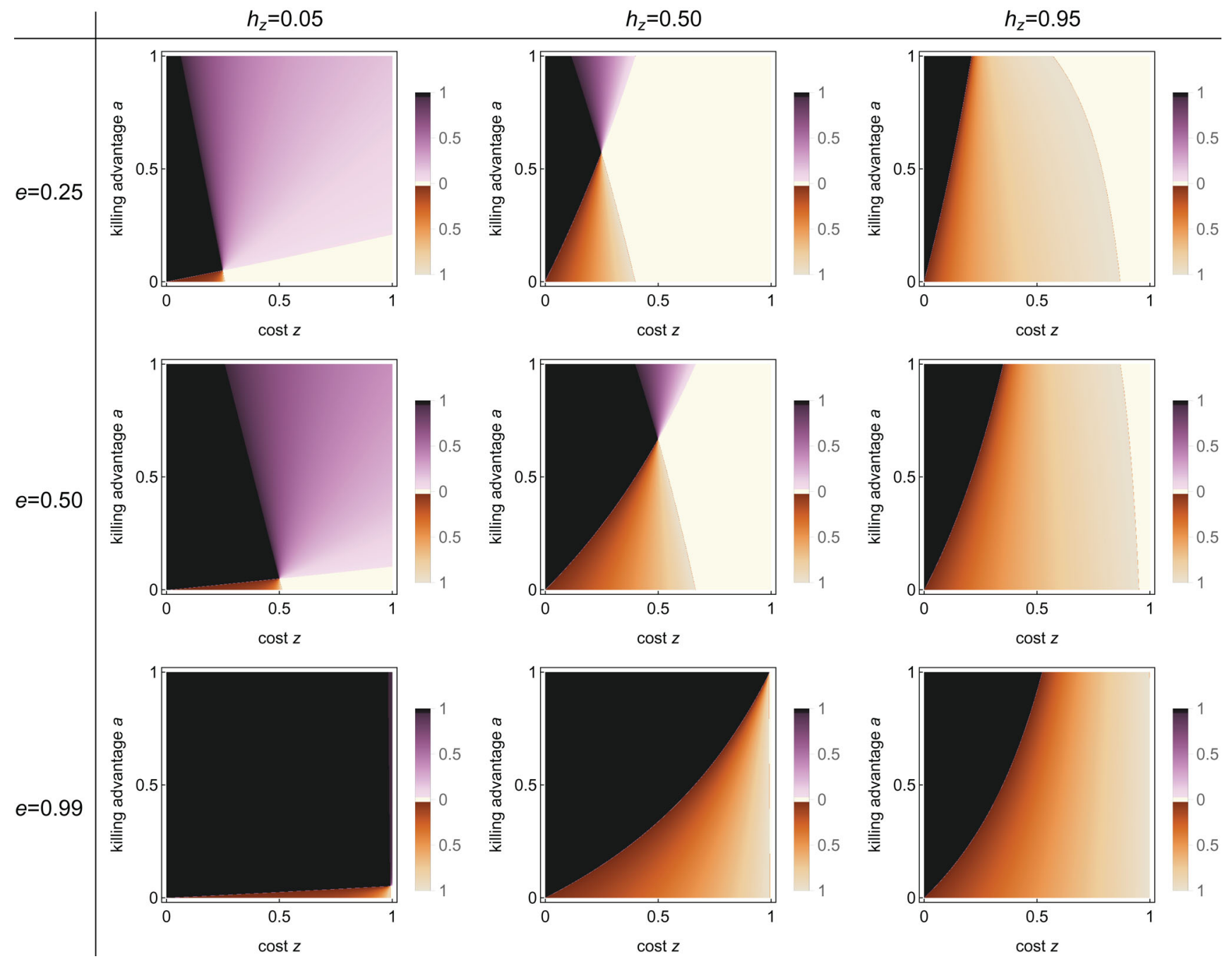

Figure 3. Bifurcation analysis of the Neurospora model with diploid fitness costs $z$. The bifurcation parameters are diploid fitness costs $z$ and their dominance $h_{z}$, killing advantage $a$, and killing efficiency e. Extinction $\left(\hat{p}_{D}=0\right)$ and fixation $\left(\hat{p}_{D}=1\right)$ of the killer allele $D$ always constitute equilibria. Additionally, one interior equilibrium is possible. Parameter regions are color coded as follows: white, $D$ cannot invade and $\hat{p}_{D}=0$ is a globally stable equilibrium; black, $D$ can invade and reach fixation, $\hat{p}_{D}=1$ is a globally stable equilibrium; purple, $D$ can invade but cannot reach fixation, instead coexisting with the nonkilling allele $d$ at a globally stable interior equilibrium $0<\hat{p}_{D}<1$, whose value is given by the shade of purple; brown, the two boundary equilibria $\hat{p}_{D}=0$ and $\hat{p}_{D}=1$ are stable, with their basins of attraction separated by an unstable interior equilibrium $0<\hat{p}_{D}<1$ whose value is given by the shade of brown. Each panel is based on $1000 \times 1000$ parameter combinations. Gametic fitness costs $g=0$.

\section{Results}

\section{Neurospora}

\section{Deterministic model}

The dynamics of the spore-killing allele D in Neurospora, as described by equation (1), can be analyzed analytically. Solving this equation for its equilibria, we obtain $\hat{p}_{D}=0, \hat{p}_{D}=1$, and

$$
\hat{p}_{D}=\frac{1-L_{D}}{1-L_{D}+L_{D D}-L_{d}} .
$$

This last equilibrium is valid (i.e., $0<\hat{p}_{D}<1$ ) if $L_{D}<1$ and $L_{D D}>L_{d}$, or if $L_{D}>1$ and $L_{D D}<L_{d}$. Next, we discuss how the conditions for invasion, fixation and coexistence of the killer allele $D$ are affected by its killing efficiency, the killing advantage, and fitness costs and their degree of dominance. Figure 3 summarizes these results for the case of diploid fitness costs $z$ while the results for the case of haploid costs $g$ are shown in Figure S1.

A linear stability analysis shows that the killing allele $D$ can invade ( $\hat{p}_{D}=0$ unstable) if $L_{D}>1$, that is, if the fitness of the $D$ allele in heterozygotes exceeds the fitness of $d d$ homozygotes. This makes intuitive sense, because, as long as the $D$ allele is rare, 
it primarily occurs in heterozygous form. From equation (2b), we can see that this condition is met when

$$
\left(1-z h_{z}\right)(1-g)(1+a e)>1 \text {. }
$$

Thus, invasion is possible if the fitness costs to a $D$ allele (i.e., $\left.\left(1-z h_{z}\right)(1-g)\right)$ are more than outweighed by an increase in fitness due to the realized killing advantage (i.e., $(1+a e))$. In particular, invasion is more likely the more recessive the cost $z$ (decreasing $h_{z}$, compare columns in Fig. 3). In the absence of costs $(g=0, z=0)$, or in the presence of only fully recessive diploid costs $\left(h_{z}=0\right)$, invasion is possible if $a e>0$.

In the absence of both costs ( $g=0$ and $z=0$ or $\left.h_{z}=0\right)$ and killing advantage $(a=0), L_{D}=1$, resulting in equality in condition (10). In this case, a second-order stability analysis (Otto and Day 2011, p. 166) shows that invasion is possible if $e>0$. Thus, a simple spore killer without fitness costs and killing advantage can still invade. However, under these conditions, a spore killer is a purely relative drive; its proportionate rate of increase depends positively on its frequency (Appendix) and, in particular, is very small when the spore killer is rare (Nauta and Hoekstra 1993; Appendix). Therefore, invasion of the spore killer in this case is precarious, with stochastic loss a common outcome (see the "Invasion probability of a spore killer without killing advantage" section).

A linear stability analysis of the fixation equilibrium, $\hat{p}_{D}=$ 1 , shows that it is stable with respect to invasion of the $d$ allele when the fitness of $D$ in a homozygote is higher than that of $d$ in a heterozygote, $L_{D D}>L_{d}$. Based on equations (2a) and (2c), this is the case when

$$
(1-z)(1-g)>\left(1-z h_{z}\right)(1-e)(1+a e) .
$$

From this inequality, we can see that fixation becomes less likely with increasing costs $g$ and $z$. Furthermore, fixation becomes also less likely with a decreasing dominance parameter $h_{z}=0$, increasing killing advantage $a$ and decreasing killing efficiency $e$, which all favor the $d$ allele when the frequency of $D$ is high.

The two conditions for invasion and fixation lead to four possible outcomes (Fig. 3). First, if $L_{D} \geq 1$ and $L_{D D}>L_{d}$, then the spore killer can invade and reach fixation, $\hat{p}_{D}=1$ is a globally stable equilibrium. Second, if $L_{D}<1$ and $L_{D D}<L_{d}$, the spore killer cannot invade and $\hat{p}_{D}=0$ is a globally stable equilibrium. Third, if $L_{D} \geq 1$ and $L_{D D}<L_{d}$, the spore killer can invade and coexist with the sensitive allele in a stable polymorphism. Fourth, if $L_{D}<1$ and $L_{D D}>L_{d}$, the spore killer cannot invade from low frequencies, but it can reach fixation when starting from a frequency larger than the equilibrium value given by equation (9).

The conditions for stable coexistence, $L_{D} \geq 1$ and $L_{D D}<$ $L_{d}$, impose the following requirements on the model parameters. First, killing has to be incomplete $(e<1)$, else fixation of the killer cannot be stopped whenever invasion is possible. Second, some fitness costs have to exist $(z>0$ or $g>0)$, because otherwise with $e>0$ the condition for fixation would be fulfilled (recall that $a<1$ ). From inequalities (10) and (11) we can also see that a higher recessivity of the diploid costs, that is, lower $h_{z}$, favors invasion but disfavors fixation of $D$, and thus facilitates coexistence (Fig. 3). Stable coexistence is also possible under an additive diploid fitness cost provided that the killing advantage $a$ is sufficiently large (purple regions in the second column in Fig. 3).

It is insightful to discuss these results in the context of frequency-dependent selection. A killing advantage $a$ favors both $D$ and $d$ alleles when rare (see conditions 10 and 11), thus inducing a type of negative frequency-dependent selection (not in the sense that the fitnesses $L_{D D}, L_{D}$, and $L_{d}$ depend on allelic frequencies, but in the sense that the strength and direction of selection acting on the individual alleles depends on their frequencies) that can favor coexistence of the two alleles. The reason is that killing advantage only occurs in asci deriving from heterozygotes (see Fig. 2), which applies more often to the rarer allele. There is, however, an important distinction between the benefits that $D$ and $d$ can obtain from killing advantage when rare. The sensitive allele $d$ is the victim of killing and only benefits from killing advantage when killing is incomplete $(e<1$, see condition 11). Bistability, the situation in which both boundary equilibria are stable and separated by an interior unstable equilibrium, requires that each allele has a disadvantage when rare (positive frequency-dependent selection). The $d$ allele is disfavored when rare because the risk of being killed in an ascus increases with increasing frequency of $D$. On the other hand, the $D$ allele is disfavored when rare if the zygotic costs $z$ are sufficiently dominant ( $h_{z}$ sufficiently large). The magnitude of the killing advantage and the cost parameters determine whether the forces of negative or positive frequency-dependent selection dominate.

In summary, in the Neurospora model: (i) A spore killer can invade if it bears no fitness costs, or if the costs are out-weighed by a killing advantage. (ii) If the killer allele can invade, then stable coexistence with the sensitive allele is possible provided that killing is incomplete $(e<1)$ and there are sufficiently strong fitness costs to the spore killer, preferably recessive, or a strong killing advantage that benefits the sensitive allele in spores surviving killing.

\section{Invasion probability}

Our stochastic simulations show that invasion of the killing allele $D$ is possible whenever the equilibrium $\hat{p}_{D}=0$ is unstable. For diploid costs this is shown in Figure 4 (see Fig. S2 for haploid costs). The probability of invasion increases as costs and their dominance decrease. Furthermore, when the spore killer is associated with a killing advantage, fitness costs, or both, the de- 


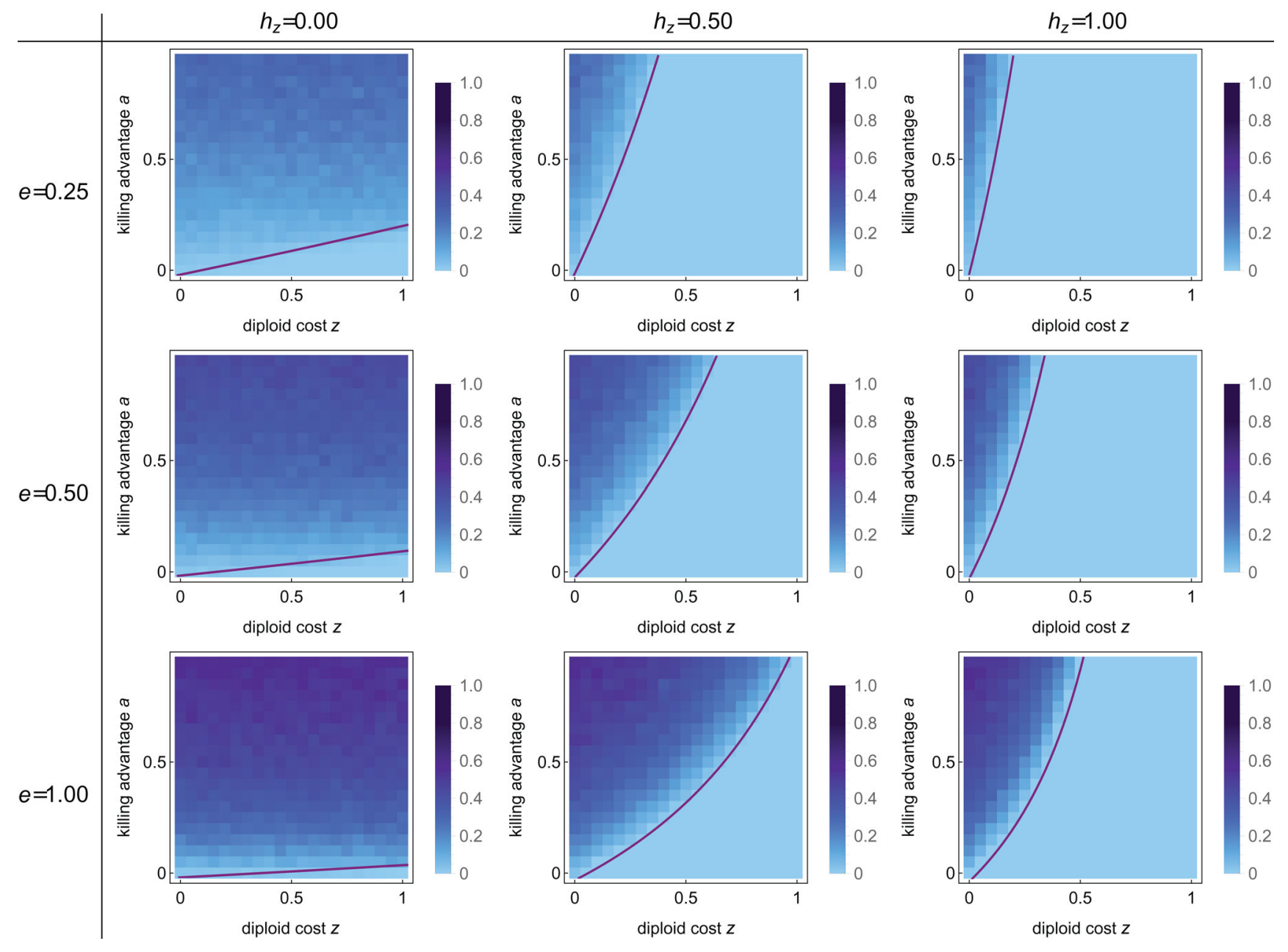

Figure 4. Invasion probability of a spore killer in the Neurospora model with diploid fitness costs $z$. Parameters are the fitness costs $z$ and their dominance $h_{z}$, the killing advantage $a$, and the killing efficiency e. Each panel consists of $21 \times 21$ parameter combinations and shades of blue indicate the invasion probability estimated from $10^{3}$ simulations of a Wright-Fisher process with population size 1000. Parameter combinations above and to the left of the purple line allow for invasion of the spore killer according to the deterministic model. Other parameters as in Figure 3.

pendency of the invasion probability on population size becomes negligible (see Fig. S20). The special case with neither fitness costs nor killing advantage is treated in the "Invasion probability of a spore killer without killing advantage" section.

\section{Podospora anserina}

\section{Deterministic model}

The dynamics of the spore-killing allele $D$ in the $P$. anserina model are affected by all parameters listed in the legend of Figure 2, and a complete analysis of all parameter combinations is beyond the scope of this article. In the following, we analyze this model in two steps. In the first step we focus on the simplified $P$. anserina model described in the "Recursion equation for a simplified Podospora model" section. This allows us to analytically investigate the effects of FDS and SDS and of the dikaryotic costs $k$. In the second step, we analyze the complete model, which ad- ditionally includes selfing with probability $s$, monokaryons with probability $m$, costs associated to monokaryons $k_{m}$, and inbreeding costs $i$. For this second step, we rely on parameter sweeps. Table 1 summarizes the examined parameter combinations and the corresponding figures.

\section{The simplified Podospora model}

As the simplified Podospora model follows the same recursion equation as the Neurospora model (eq. 1), but with modified fitness values (compare eqs. 2 and 3 ), the two models have the same equilibrium structure. The first column in Figure 5 shows how the equilibria are affected by the killing advantage $a$, the probability of $\operatorname{FDS} f$, and a recessive diploid fitness cost $z$. Results for other levels of dominance, dikaryotic cost $k$ at different levels of dominance and gametic cost $g$ are shown in the first column of the corresponding figures as indicated in Table 1. 


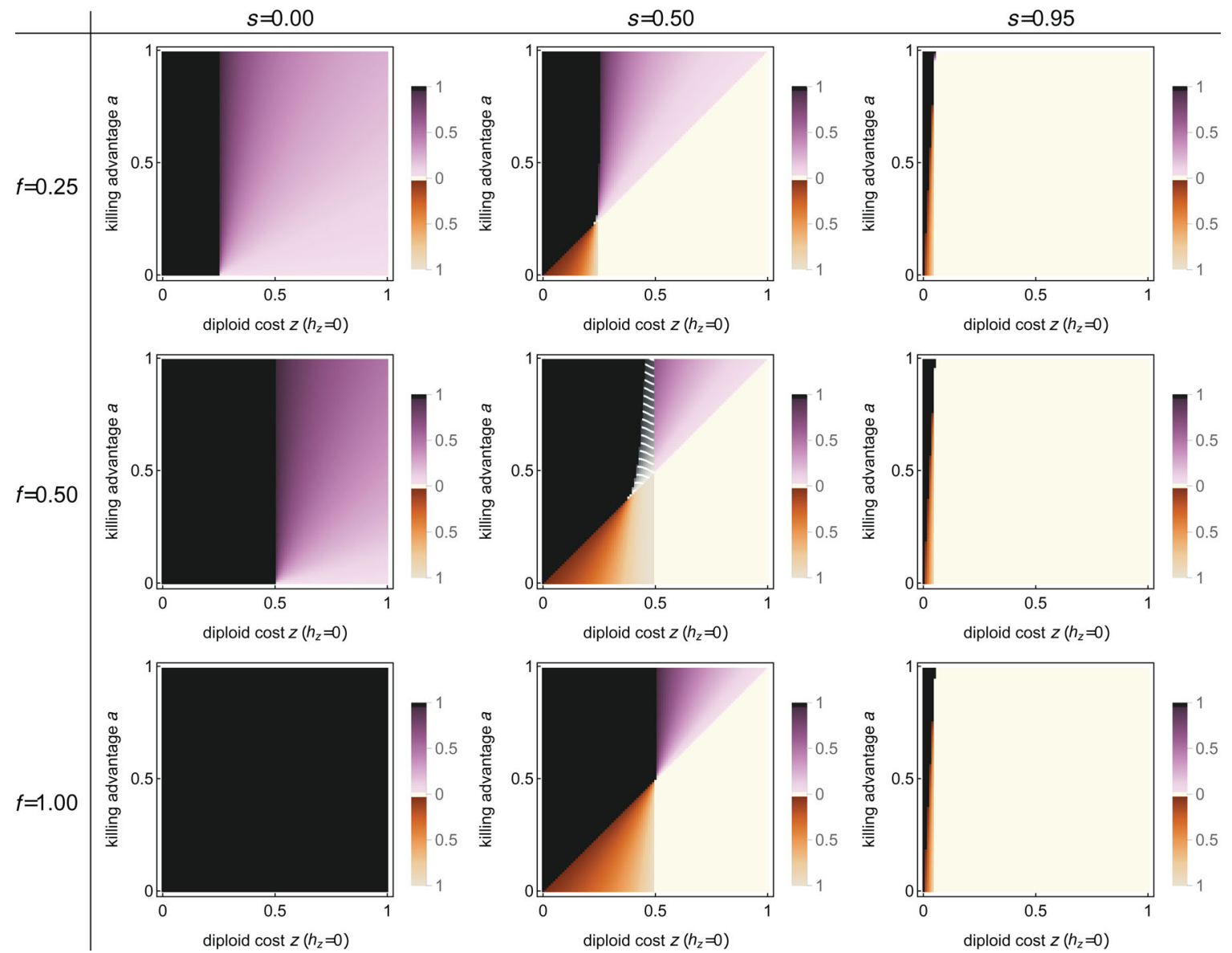

Figure 5. Bifurcation analysis of the Podospora model with recessive $\left(h_{z}=0\right)$ diploid fitness costs $z$. The bifurcation parameters are diploid fitness costs $z$, killing advantage $a$, selfing rate $s$, and probability of the first-division segregation $f$. Extinction $\left(\hat{p}_{D}=0\right)$ and fixation $\left(\hat{p}_{D}=1\right)$ of the killer allele $D$ always constitute equilibria. Additionally, one or two interior equilibria are possible. Parameter regions are color coded as follows: white, $D$ cannot invade and $\hat{p}_{D}=0$ is a globally stable equilibrium; black, $D$ can invade and reach fixation, $\hat{p}_{D}=1$ is a globally stable equilibrium; purple, $D$ can invade but cannot reach fixation and coexists with the nonkilling allele $d$ at a globally stable interior equilibrium $0<\hat{p}_{D}<1$ whose value is given by the shade of purple; brown, the two boundary equilibria $\hat{p}_{D}=0$ and $\hat{p}_{D}=1$ are stable, with their basins of attraction separated by an unstable interior equilibrium $0<\hat{p}_{D}<1$ whose value is given by the shade of brown; gray with white stripes, two interior equilibria exist, the equilibrium with the lower value being stable, meaning that $D$ can invade and coexist with $d$ at a stable interior equilibrium, whose value is given by the shade of gray. Each panel is based on $100 \times 100$ parameter combinations. Other fixed parameters: $e=1, m=0$, other fitness costs are zero.

For the same reason as discussed for the case of Neurospora (c.f. eq. 10), the killing allele $D$ can invade if $L_{D}>1$. This is the case when

$$
\left(1-z h_{z}\right)(1-g)\left(f(1+a e)(1-k)+(1-f)\left(1-k h_{k}\right)\right)>1 .
$$

As for the Neurospora model, invasion of the $D$ allele is possible if the realized killing advantage $(1+a e)$ outweighs the fitness costs $z, g$, and $k$ that occur at different stages during the life cycle, with the last one being specific to Podospora.

In Podospora, because spores are dikaryotic, the segregation of the killer locus during meiosis matters and is accounted for by the parameter $f$ describing the probability of FDS. Different out- comes are possible for the invading allele depending on whether FDS occurs (probabilities $f$ and $1-f$, respectively). Under FDS the packaging of nuclei into homoallelic spores leads to killing, which provides the $D$ allele with a killing advantage $a e$, but also exposes it to the full dikaryotic cost $k$. Alternatively, if FDS does not occur (resulting in the formation of heteroallelic spores), $D$ is deprived of a killing advantage but may only suffer a reduced (unless fully dominant) dikaryotic cost $k h_{k}$. Generally, the killer will benefit from increasing probabilities of FDS as long as the condition $(1+a e)(1-k)>\left(1-k h_{k}\right)$ is fulfilled, which is the case whenever invasion is possible.

The effect of the dikaryotic cost $k$ on invasion thus depends on $f$ if $h_{k}<1$. By contrast, the diploid cost, which only appears 
Table 1. Overview of the parameter combinations investigated in the Podospora model.

Figure

\begin{tabular}{cccccc}
$m$ & $e$ & Cost & Dominance & Deterministic & Stochastic \\
\hline 0 & 1 & $z$ & $h_{z}=0$ & Figure 5 & Figure S13 \\
& & & $h_{z}=0.5$ & Figure S3 & Figure S14 \\
& & & $h_{z}=1$ & Figure S4 & Figure S15 \\
& & $k$ & $h_{k}=0$ & Figure S5 & Figure S16 \\
& & & $h_{k}=0.5$ & Figure S6 & Figure S17 \\
& & & $h_{k}=1$ & Figure S7 & Figure S18 \\
& & $g$ & & Figure S8 & Figure S19 \\
& 0.8 & $z$ & $h_{z}=0$ & Figure S9 & - \\
& & & $h_{z}=0.5$ & S10 & - \\
\hline 0.05 & 1 & $z$ & $h_{z}=0$ & S11 & - \\
\hline 0.5 & & & & S12 & - \\
\hline
\end{tabular}

in the form $z h_{z}$ because $D$ is exclusively heterozygous during invasion, and the gametic cost $g$ occur independent of $f$. This distinction between the diploid and dikayrotic costs arises because the diploid cost is expressed before meiosis and the dikaryotic cost afterwards (see life cycle in Fig. 2). For this reason, during invasion the diploid cost is expressed exclusively in the heterozygous form, while the dikaryotic cost is expressed in the homozygous form whenever FDS (and therefore killing) occurs. As a result, the dikaryotic cost can be a much stronger counteracting force to the invasion of the killer than the diploid cost, at least as long as it is not fully dominant. The effect of dikaryotic and diploid costs on the possibility for the $D$-allele to invade can be seen when comparing Figure 5 with Figure S5 and Figure S3 with Figure S6. We can see that the dikaryotic cost affects the killer more strongly by preventing its invasion at lower cost magnitudes, and that the difference between the two types of costs is more marked the more recessive they are. When fully dominant, their effects are identical.

Finally, we highlight two special cases regarding the condition for invasion. First, with a fully recessive diploid fitness cost $\left(h_{z}=0\right)$ and in the absence of gametic and dikaryotic costs, invasion is always possible as long as $a>0$ (see first columns in Fig. 5). Second, the absence of both a killing advantage $(a=0)$ and costs $(z=k=g=0)$ represents the same special case of a purely relative drive as described for the Neurospora model for which invasion is possible as long as $e>0$.

Close to fixation of the $D$ allele, the $d$ allele is rare and occurs almost exclusively in the heterozygous form. Thus, $D$ reaches fixation when the fitness of $D$ in a $D D$ homozygote exceeds the fitness of $d$ in a $D d$ heterozygote, $L_{D D}>L_{d}$. This is the case when

$$
(1-z)(1-k)(1-g)>\left(1-z h_{z}\right)(f(1+a e)(1-e)
$$

$$
\left.+(1-f)\left(1-k h_{k}\right)\right)
$$

As for invasion, the probability of FDS only matters for the rare allele, now $d$, for which segregation at meiosis can lead to different outcomes. From the right-hand side of inequality (13) it is clear that FDS favors fixation of $D$ if $(1+a e)(1-e)<$ $\left(1-k h_{k}\right)$, that is, if the fitness cost due to spore killing, potentially partially compensated by a killing advantage, is less than the dikaryotic cost in heterozygous form. This condition is always fulfilled in the case of complete killing $(e=1)$, or fully recessive dikaryotic cost $\left(h_{k}=0\right)$ or absence of that cost $(k=0)$. This finding is confirmed by the first column of Figures 5 and $\mathrm{S} 3-\mathrm{S} 5$, in which $e=1$, and Figures $\mathrm{S} 9$ and $\mathrm{S} 10$, in which $k=0$.

One consequence of incomplete FDS $(f<1)$ is that coexistence of the alleles $D$ and $d$ becomes possible even in the presence of complete killing ( $e=1$ ), while in the Neurospora model complete killing always results in the fixation of the $D$ allele whenever invasion is possible. Coexistence under complete killing requires that the $D$ allele suffers a recessive cost $\left(h_{z}<1 / 2\right.$ or $\left.h_{k}<1 / 2\right)$. For example, for the case of a fully recessive diploid cost $\left(h_{z}=0\right)$ and in the absence of other costs, the condition for invasion, inequality (12), simplifies to $f a>0$ and the one for fixation, inequality (13), to $f>z$. Coexistence is then possible with $e=1$ as long as $f<z$ (see the first column in Fig. 5). Finally, we note that fully recessive dikaryotic costs $\left(h_{k}=0\right)$ affect the condition for fixation in the same manner as gametic costs (compare the first columns of Figs. S5 and S8).

\section{The complete Podospora model}

Compared to the simplified Podospora model, the complete model includes the possibility for selfing and the formation of monokaryons, and it is these two aspects on which we focus in this section. We note that the complete Podospora model allows for the existence of two internal equilibria, the lower of which is stable, and the upper unstable. However, this case occurs only in a small region of the parameter space (Fig. 5, dashed area).

(i) Effect of selfing. Here, we explore the effect of selfing on the dynamics of the spore killer allele $D$. We start by focusing on the case of complete killing $(e=1)$, no monokaryons $(m=0)$, and fully recessive diploid cost $\left(h_{z}=0\right)$, and subsequently relax these restrictions in sections (ii) and (iii). Given these restrictions, the second and third columns in Figure 5 show the effect of an intermediate and a high selfing probability, respectively. For these findings we can provide intuitive explanations but no analytical proof. The following observations can be made.

First, invasion of the $D$ allele becomes more difficult with increasing selfing probability. For instance, for the parameters used in Figure 5 and with $s=0.5$, invasion becomes 
impossible if the diploid cost exceeds the killing advantage $(z>a)$, whereas invasion is always possible with $s=0$ (compare first and second column in Fig. 5). For higher selfing probabilities invasion is restricted to even smaller regions of the parameter space. The reason is that $D: D$ dikaryons that reproduce by selfing result in $D D$ diploids and these pay the full diploid fitness cost. Random mating instead produces mostly $D d$ diploids during invasion due to the low frequency of $D$. The reduction of the parameter space allowing for invasion of the $D$ allele due to this mechanisms is more pronounced the more recessive the diploid costs are (lower $h_{z}$, compare the second column in Figs. 5, S3, and S4).

Second, fixation of the $D$ allele generally becomes more difficult with increasing selfing probability. The reason is that selfing maintains $d: d$ dikaryons and thereby protects the $d$ allele from spore killing. However, this effect of selfing on the fixation of $D$ becomes visible only at sufficiently low levels of SDS because high levels of SDS also shield $d$ from spore killing. For instance, Figure 5 shows that increasing the selfing probability from $s=0$ to $s=0.5$ does not affect fixation of the $D$ allele at low $(f=0.25)$ or moderate $(f=0.5)$ levels of FDS but does hinder fixation at high levels $(f=1)$. Qualitatively, similar observations hold for additive and dominant diploid costs (Fig. S3 and S4). This effect is further modulated by the level of dominance of the dikaryotic costs. These costs decrease the effectiveness of SDS in protecting the $d$ allele, which gives SDS a comparatively smaller role in preventing fixation of $D$ with increasing $h_{k}$ (compare Fig. S5 with Figs. S6 and S7).

We note that selfing can involve a fitness cost $i$ due to inbreeding at all loci, which applies to individuals regardless of their genotype. This cost reduces the effective selfing rate by removing a portion of selfing individuals and does not otherwise interact with the dynamics of the spore killer.

(ii) Effect of incomplete killing. We restrict ourselves to the case of diploid costs $z$, which are more conducive to invasion of the killing allele than dikaryotic and gametic costs. Figure S9 shows a bifurcation diagram analogous to Figure 5 but with $e=0.8$. As expected, lowering the killing efficiency lowers the upper boundaries of the diploid costs below which invasion and fixation of the spore killer are possible. This observation also holds under additive costs (compare Figs. S3 and S10). As can be seen, incomplete killing $(e<1)$ enables coexistence in the presence of an additive diploid fitness cost.

(iii) Effect of monokaryons. A final aspect of the P. anserina life cycle that we explore is the effect of monokaryons on the dynamics of the spore killer. As can be seen from Figure 2, monokaryons are not able to self and they allow for a small amount of spore killing even in the case of SDS. Thus, by limiting the selfing rate and by allowing killing even when the probability of FDS is low we expect that monokaryons facilitate both invasion and fixation of the spore killer.

For the case of recessive diploid costs $\left(h_{z}=0\right)$ and no other costs we can formalize the effect of monokaryons as follows. Based on the life cycle in Figure 2, we can express the proportion of killed spores, $K$, as

$$
K=P_{D d} e\left(\frac{f}{2}+\frac{m}{4} \frac{(1-f)}{2}\right) .
$$

Thus, the amount of killed spores increases with the frequency of monokaryons $m$, proportionally to the probability of SDS events at meiosis $(1-f)$, and with the frequency $P_{D d}$ of heterozygotes in the population (which itself increases with $m$ due to outcrossing).

For $5 \%$ and $50 \%$ of asci containing a pair of monokaryons, Figures S11 and S12, respectively, show how monokaryons increase the upper boundaries of the diploid costs below which invasion and fixation of the spore killer are possible. These figures should be compared to Figure 5, which corresponds to the same parameter values but without monokaryons. A frequency of $5 \%$ is in the range expected in natural populations, but we also consider $50 \%$ to magnify the effect of monokaryons. In the case of $50 \%$ of asci containing monokaryons, the effects become clearly visible (Fig. S12). With $5 \%$ of asci containing monokaryons, their effect becomes negligible, indicating that they probably do not greatly affect spore killer dynamics under natural conditions.

The results for the Podospora model can be summarized as follows. (i) A spore killer can invade if it bears no fitness costs, or if the costs are outweighed by the fitness benefit due to a killing advantage. (ii) Selfing makes invasion and fixation of the spore killer more difficult. This is because it reduces the frequency of heterozygotes and increases the chance that recessive costs become expressed. Selfing interacts with the probability of FDS to determine the effective killing rate. (iii) Coexistence between a spore killer and a sensitive allele is possible with incomplete killing efficiency $(e<1)$ in combination with recessive or additive fitness cost, and remains possible with fully efficient killing $(e=1)$ in combination with a recessive fitness cost and either some amount of selfing or $\operatorname{SDS}(f<1)$.

\section{Invasion probability}

Our stochastic simulations confirm that invasion of the killing allele $D$ is possible whenever the deterministic equilibrium $\hat{p}_{D}=0$ is unstable. The probability of invasion increases with the probability of FDS $f$ and the killing advantage $a$, which both increase 
A

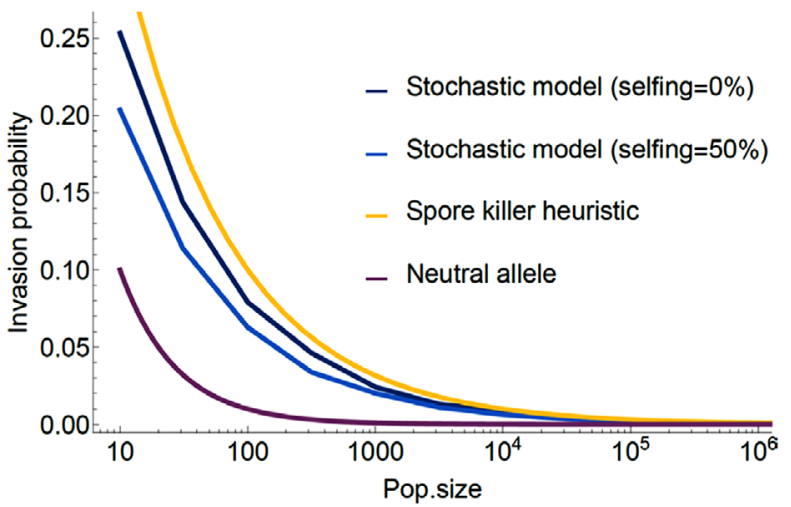

B

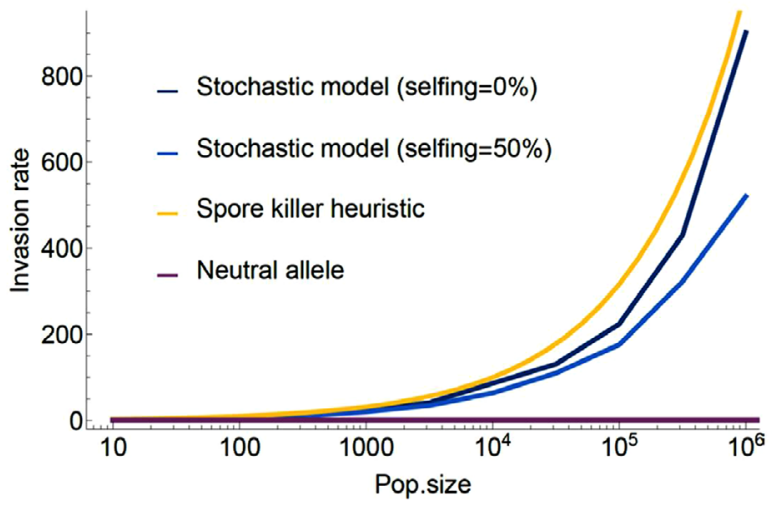

Figure 6. Invasion probability (A) and invasion rate (B) of a spore killer as a function of population size. Fitness costs or killing advantage are absent. A comparison is made between the approximation developed in the "Invasion probability of a spore killer without killing advantage" section (spore killer heuristic), the stochastic invasion model, and the stochastic invasion model with $50 \%$ selfing rate. A neutral allele is also represented for reference.

the selective advantage of the spore killer. In turn, the invasion probability decreases with fitness costs. We refer the reader to Table 1 for an overview of the parameter combinations investigated and the corresponding figures.

\section{INVASION PROBABILITY OF A SPORE KILLER WITHOUT KILLING ADVANTAGE}

A spore killer without killing advantage is a purely relative drive, and consequently its invasion probability tends to zero as the population size $N$ becomes large (Nauta and Hoekstra 1993). However, it has not been investigated how rapidly this invasion probability declines as $N$ grows, and how the contrasting effects of population size on invasion probability and mutational supply interact to determine the overall rate of invasion of spore killers.

\section{Invasion probability as a function of population size $N$}

We study the simplest possible model of a newly arisen spore killer, assuming random mating (no selfing). This model corresponds to the Neurospora model with all fitness costs set to 0 , $a=0$, and $e=1$. Employing a heuristic method from Desai and Fisher (2007, p. 1763), we show in Appendix that, once the spore killer exceeds $n=\sqrt{N}$ copies, the expected increase in its copy number over the subsequent $n$ generations $\left(\sim n^{3} / N\right)$ is greater than typical decreases in copy number by random drift over the same period $(\sim n)$, so that the spore killer has very likely escaped stochastic loss. The dynamics of the spore killer before reaching $\sqrt{N}$ copies are dominated by random drift, so the probability that it attains the required $\sqrt{N}$ copies-that is, its invasion probability-is approximately $1 / \sqrt{N}$ (Crow and Kimura 1970). This approximation agrees well with estimates obtained from simulations (Fig. 6A).

\section{Invasion rate as a function of population size $N$}

If spore killers arise at a rate $\mu$ per replication, then their rate of appearance per generation is $N \mu$. Therefore, the per-generation rate of invasion of such spore killers is $N \mu \times 1 / \sqrt{N}=\sqrt{N} \mu$. The invasion rate is thus an increasing function of population size (Fig. 6B), despite the fact that the invasion probability of each individual spore killer decreases as $N$ increases, tending to zero in the large-population limit. Additional life-history features, such as selfing, affect the invasion rate but do not affect the positive scaling with population size (Fig. 6B).

\section{Invasion probability and population structure}

The dependence of the invasion probability on population size suggests that population structure could significantly affect the rate at which spore killers invade. Suppose that the population of size $N$ is subdivided into $M$ demes, each of size $m=N / M$. The overall arrival rate of new spore killers is unchanged $(N \mu)$, but, because invasion of one deme guarantees population-wide invasion (unless $m$ is very small), the invasion probability is $1 / \sqrt{m}$. The invasion rate is therefore $N \mu \times 1 / \sqrt{m}=\mu \sqrt{M} \sqrt{N}$, which is larger than in an unstructured population by a factor of $\sqrt{M}$. A similar result has been obtained for recessive beneficial mutations (Gale 1990, pp. 180-181), which are under similar frequencydependent selection (see Appendix).

\section{Discussion}

Our study is novel in exploring the effect of several aspects of fungal life cycles on the conditions under which a sporekilling allele can invade and subsequently stably coexist with a non-killing allele. In ascomycete fungi, spore killing takes place within the ascus, and our model is based on a detailed 
mechanistic understanding of ascus composition (see Fig. 2). Our results show that following the possible compositions of spores within an ascus is necessary to fully understand the dynamics of a spore-killing allele. Another novel aspect of our study is the development of stochastic models to investigate the invasion probability of a spore-killing allele, complementing our deterministic analysis. Our model is based on a single allele responsible for both spore killing and resistance to spore killing. Thus, there is no recombination between the two functions. This feature is consistent with the picture emerging from recent genetic characterizations of spore killers in several species of ascomycete fungi (Hu et al. 2017; Nuckolls et al. 2017; Vogan et al. 2019; Svedberg et al. 2020). The only other theoretical study of spore killing we are aware of (Nauta and Hoekstra 1993) focuses on the role of recombination between the killing and resistance functions, which was appropriate given what was known about the genetic architecture of spore killers at that time in Neurospora, but appears now to be the exception rather than the rule.

In the following, we first discuss the general insights that our model reveals about the dynamics of spore killers. We then discuss our results in the light of data from natural systems, with particular attention paid to the spore killer systems $S p k-1$ in Neurospora and Spok in Podopsora, which inspired our model. Throughout, we compare our findings to theoretical and empirical results from male and female MDs in animals and plants.

\section{SPORE KILLERS IN THEORY}

\section{Selective advantage and invasion of a spore killer}

We start by comparing the dynamics of spore killers in our model with Hartl's (1972) general model of sperm and pollen killers. In Hartl's model, gamete killers kill in the homozygote form, which could be seen as "self-killing" or "suicide" as effectively the killer allele eliminates other copies of itself. This "self-killing" does not provide any fitness advantage for the killer in the absence of a killing advantage. In contrast, spore killers do not "self-kill", which allows them to obtain a positively frequency-dependent selective advantage simply by killing. In agreement with Nauta and Hoekstra (1993), we find that this frequency-dependent selective advantage tends to be 0 when the frequency of the spore killer is close to 0 . Thus, in the absence of any killing advantage, the selective advantage of a spore killer is minimal at the onset of invasion (except in small populations), and so invasion of spore killers can be prevented by any non-recessive fitness costs, or simply by stochastic loss (see the "Invasion probability of a spore killer without killing advantage" section). This distinguishes spore killers from female drive systems, in which the selective advantage induced by drive itself can be substantial at all frequencies of the driver, allowing female drivers to invade even when they are associated with substantial fitness costs (e.g., Hall and Dawe 2018).
Although the invasion probability of a spore killer without killing advantage is substantially lower than that of a female driver, it is not negligible ("Invasion probability of a spore killer without killing advantage" section). Moreover, owing to the frequency-dependent nature of the spore killer's selective advantage, small or fragmented populations may represent easier targets for invasion. Thus, spore killers in structured populations, which we have only briefly addressed in the "Invasion probability of a spore killer without killing advantage" section, merit further study. In addition, our calculations show that, even in the absence of a killing advantage, the invasion rate of spore killers increases with population size, as the increase in mutational supply of spore killers more than compensates for the decrease in their individual invasion probabilities ("Invasion probability of a spore killer without killing advantage" section). This last point depends, of course, on the mechanism of origin of spore killers, which we discuss in the next section.

In addition to the action of killing itself, we propose that spore killers could induce a killing advantage, that is, a net fitness benefit from killing, either in the form of compensation or reduced local competition for resources. In the model of Hartl (1972), compensation plays a crucial role for gamete killers, granting them a selective advantage. Although we cannot draw a direct parallel to Hartl's model, which focuses on the link between gamete number and fecundity, we find that a killing advantage is also crucial for the invasion of a spore killer, conferring a nonnegligible selective advantage even when the spore killer is rare, and can therefore promote its initial invasion. In particular, a killing advantage reduces the chance of stochastic loss of a killing allele. It is therefore vital, in future empirical studies of spore killer systems, to determine whether and to what extent a killing advantage is present.

\section{Coexistence of a spore-killing allele with a nonkilling allele}

We also investigate the conditions for stable coexistence of the killing and nonkilling alleles. MDs are expected to spread rapidly to fixation (Lindholm et al. 2016), rather than coexisting with their nondriver alleles in a stable polymorphism - and when an MD is fixed, drive does not occur, rendering the MD undetectable. The MDs that are observed in natural populations are therefore expected to exhibit (possibly unusual) properties that promote their maintainance in stable polymorphisms. Understanding these properties is therefore an important task of theoretical studies of meiotic drive. Classically, in male and female drive, recessive fitness costs associated with the driving allele are required for coexistence (e.g., Lewontin and Dunn 1960; Hartl 1970; Fishman and Kelly 2015; Holman et al. 2015)_invasion is permitted because the costs are not expressed in heterozygotes, while fixation is prevented because the costs are expressed in 
homozygotes. We find these dynamics in our models for both Neurospora and Podospora. Fitness costs are needed for coexistence to be possible, and recessive costs increase the parameter space that supports coexistence (Fig. 5). For coexistence to be possible, it is also necessary that killing is incomplete, so that the sensitive allele obtains a fitness benefit when in a diploid heterozygote. Incomplete killing can result from the killing efficiency being less than $100 \%$ and, in the case of Podospora, from SDS.

Intriguingly, we find that coexistence may also be possible even if fitness costs to the spore killer are not recessive. Such coexistence occurs because sensitive spores that survive killing also benefit from the killing advantage. We believe that this assumption is empirically reasonable given the two possible scenarios that we envision underlying a killing advantage, namely compensation and reduced local competition. Compensation results in additional spores-both killer and sensitive-being produced by the parent in response to killing. If a killing advantage occurs via reduced local competition with siblings, then the same reasoning applies and both types of surviving spores obtain a fitness advantage. Coexistence can occur in this case because the benefit to the surviving sensitive spores prevents fixation of the spore killer by raising the fitness of the sensitive allele in heterozygotes above the fitness of the spore killer in homozygotes. Thus, incomplete killing can result in coexistence just as recessive costs can (or overdominance, Hartl 1970), but the underlying biology is distinct.

\section{Mating system and spore killer dynamics}

We find that the rate of selfing of the host has a negative effect on the invasion of a spore killer. The reason is that selfing decreases the frequency of heterozygotes (in which spore killing occurs), and magnifies potential fitness costs, generally slowing down invasion. Because of this latter point, our model predicts that a spore killer can invade a population with a high selfing rate only when it is associated with low fitness costs. Moreover, coexistence is then unlikely. We expect inbreeding to have the same effect as selfing, and analyzing spore killer models in which the assumption of random mating is relaxed could be an interesting next step. This influence of selfing further suggests that mating behavior itself could evolve as a defense against spore killers, as previously suggested by Lewontin and Dunn (1960). Along the same lines, Bull (2017) and Bull et al. (2019) have developed models showing that inbreeding can evolve as an efficient response to costly MDs, while Burt and Trivers (1998) have shown that obligatorily outcrossing plant species are more susceptible to costly selfish genetic elements.

\section{INSIGHTS FROM NATURAL SYSTEMS}

\section{How much do we know about spore killers in nature?}

In several model systems of male and female drive, the molecular mechanism of the MD, its fitness effects, and the biology of the host are known to a sufficient extent that population genetics models can predict the frequency of the MD in natural or laboratory populations with impressive accuracy (e.g., Lewontin and Dunn 1960; Fishman and Kelly 2015). In the case of spore killers, however, although several recent publications have shed light on the genetic and molecular basis of their driving action (Hu et al. 2017; Nuckolls et al. 2017; Vogan et al. 2019; Svedberg et al. 2020), many unknowns remain, particularly regarding the ecology of the hosts. In this section, we summarize the available knowledge and use it to put our results in perspective and to suggest future directions for empirical research.

We focus on the three spore killers in fungal hosts that are best understood: the Spok gene family in P. anserina (Vogan et al. 2019) and Spk-1 in N. sitophila (Svedberg et al. 2020)-both of which directly inspired our models - and finally the wtf gene family in Schizosaccharomyces pombe (Hu et al. 2017; Nuckolls et al. 2017), which is also well studied and has many similarities with the first two. In all three cases, spore killing and resistance are governed by a single locus, as we have assumed in our model. Although we restrict ourselves to these three bestknown example from model species, we believe that spore killers could be widespread in the fungal kingdom, or at the very least in ascomycetes where they have been observed in several taxa (Raju 1994). Fungi show extreme diversity in their mode of reproduction (Nieuwenhuis and James 2016), and our results suggest that high selfing rates may be a major factor in hampering the invasion of spore killers. Reproductive strategy may therefore be a key determinant for the presence of spore killers in a given species. Most fungi are able to reproduce sexually (Nieuwenhuis and James 2016), and are therefore susceptible to spore killers in theory, but many have high rates of selfing (Nieuwenhuis and James 2016), which our findings suggest could protect them efficiently from the invasion of spore killers.

Little is known about the origin of spore killers. It has been proposed that spore-killing systems may arise neutrally in populations in which resistance to killing has already been fixed (Sweigart et al. 2019). According to this view, spore killers would then act as a strong type of hybrid incompatibility between diverging populations. However, our work shows that an active spore killer has a substantially greater chance of invading than a neutral allele, which suggests that selfish evolution of spore killers could be more likely. The Spoks, Spk-1, and $w t f$ s all belong to large families of genes that occur across complexes of closely related fungal taxa. This suggests the possibility of horizontal gene transfer across species. For example, there is evidence that Spk-1 in N. sitophila may have introgressed from the 
closely related $N$. hispaniola (Svedberg et al. 2020). In addition to their apparently frequent movements, Spok and wtf genes mutate rapidly (Hu et al. 2017; Nuckolls et al. 2017; Vogan et al. 2019), which could be the key to their success (see the "Invasion probability of a spore killer without killing advantage" section for the importance of mutation rate).

\section{Insights from our models on spore killer dynamics in natural populations}

The Spok gene family has several members present in the genomes of species of the Podospora genus. In P. anserina in particular, three genes are known: Spok2, Spok3, and Spok4. Any given individual of $P$. anserina might carry none, one, two, or all three Spok genes. With very stable recombination rates, the FDS probability that ultimately determines the killing rate of the Spok genes is determined by their position along the chromosomes. More than one copy of a Spok gene might occur in a single genome, but this seems to be very rare (Vogan et al. 2019). Spok3 and Spok4 occur in a genomic region known as the "Spok block" (Vogan et al. 2019). The different Spok genes act independently, that is, carrying one of them does not protect against another one (Grognet et al. 2014; Vogan et al. 2019). Figure S21 shows the frequency of the three Spok genes in samples from a population of $P$. anserina near Wageningen in the Netherlands over a 17-year period (generation time can be as short as 11 days). None of the spore killers reached fixation or went extinct during this period. However, Spok2 appears close to fixation while Spok3 and Spok4 occur at lower frequencies. Additionally, all individuals without Spok2 seem to derive from a single deletion, thus, Spok2 may have been fixed prior to 1993 .

We can try to understand, with the help of predictions from our model, what characteristics of the different Spok genes may be responsible for their respective frequencies in the Wageningen population. We know that Spok2 does not kill with full efficiency and has an FDS probability of about $40 \%$, while Spok3 and Spok4 have an FDS probability ranging between $50 \%$ and $90 \%$, depending on their position in the genome (Vogan et al. 2019). Yet, Spok2 appears closer to fixation. If we take into account the presumably high selfing rate of $P$. anserina this observation is in agreement with our model, which predicts that FDS probability should matter little to the dynamics of the killer if selfing rate is high (outcrossing rate becomes limiting: compare the second and third columns in Figs. 5 and S9). Our model also predicts that a high selfing rate combined with fitness costs severely reduces the scope for invasion of a spore killer (e.g., Fig. S19). Thus, in this context, the fate of different Spok genes should be largely determined by their fitness cost but not by their FDS probability. And indeed, we know that Spok3 and Spok4 co-occur in the "Spok block" (Vogan et al. 2019), which is known to be associated with fitness costs to its host (Vogan et al. 2020). This obser- vation also confirms that increasing selfing rate may be a good defense mechanism against the invasion of spore killers, but in that context the probability of FDS, regulated by the position of the Spok genes on the chromosomes, matters little in P. anserina.

The gene Spk-1 in N. sitophila shows variation across populations, being, respectively, fixed and absent in two clades that coexist in sympatry, and polymorphic in a third clade where a form of resistance to the killer has evolved (Svedberg et al. 2020). This suggests that the dynamics of the same spore killer can vary in different populations. In the polymorphic clade, resistance has evolved in the form of reduced killing efficiency, leading to what appears to be coexistence (Svedberg et al. 2020).

Very little is known about the frequency of the wif allele in natural populations of $S$. pombe. Killing efficiency is lower than 100\% (Nuckolls et al. 2017; Núñez et al. 2020) and selfing is likely common in S. pombe (Nieuwenhuis and James 2016; Tusso et al. 2019). Furthermore, S. pombe is able to perform haploid selfing, a feature that is not found in $P$. anserina and that we therefore did not incorporate in our model. Based on this information, we predict that the fitness costs of $w t f$ must be low to allow for invasion, and that invading spore killers progress slowly and are sensitive to stochastic loss.

At least one suppressor counteracting the action of the spore killer has evolved in S. pombe (Nuckolls et al. 2017; Núñez et al. 2020). Suppressor genes are likely to evolve if given enough time (slow invasion or stable coexistence) and are found in many other drive systems, but not in P. anserina so far (Vogan et al. 2019).

The spore killers Spk-1, Spok, and wtf are all small genomic regions without inversions, suggesting that they are not necessarily associated with hitchhiking deleterious mutations (but see Vogan et al. 2020). At the same time, all spore killers function with a poison-antidote mechanism targeting spores, and it is easy to envision direct fitness costs for production and exposure of spores or even vegetative mycelium to the toxin. These costs could be recessive if subject to a threshold dosage effect. To date, there is no definitive evidence for or against fitness costs of carrying these spore killers, except for Spok3 and Spok4, which are contained within the "Spok block" element. In the latter case, it is not clear whether the cost originates from the Spok genes themselves or other features of the block. Finally, there is evidence from laboratory studies for a killing advantage in P. anserina through a recovery in the number of spores produced when killing occurs (Vogan et al. 2020). However, more work is required to understand its importance in a natural setting.

\section{CONCLUSIONS}

Despite their particularities, we predict that spore killers should show similarities with well-studied systems of meiotic drive. For example, we expect fitness costs-likely but not necessarily recessive-to explain coexistence. Our study identifies 
characteristics of the MD loci that interact with the ecology and the life cycles of ascomycete fungi and are of importance for the dynamics of spore killers. These are fitness costs, killing advantage, host population size, and the mating system. Although we find that a spore killer without costs or killing advantage is substantially more likely to invade than a neutral allele, killing advantage makes invasion substantially more likely. In contrast, selfing of the host and fitness costs associated with the killer can impede its invasion or halt its spread at intermediate frequencies.

With this work, we have explored the dynamics of spore killers in two representative species of ascomycete fungi, and have revealed novel aspects of the dynamics of these selfish genetic elements. Our work has revealed that many unknowns remain from both theoretical and empirical angles that prevent, at this stage, accurate empirical prediction of spore killer dynamics. With the advent of artificial meiotic drives, a new world of possibilities opens for biological control (Esvelt et al. 2014). If spore killers are to be used for the control of fungal pest species, there is still much work that needs to be done to fully account for their dynamics. We suggest several points of focus for future research. Important empirical tasks will be to better understand the ecology of fungal hosts, in particular regarding their mating systems, as well as to better characterize interactions between spore killers and their hosts (fitness effects, killing advantage). From a theoretical perspective, we suggest that the role of population structure and the possibility for the evolution of suppressor genes in spore killers should be important aspects.

\section{AUTHOR CONTRIBUTIONS}

IM, CR, and HJ designed the study; IM developed the theoretical model with help from CR and CV; SLAV and AV contributed with knowledge of the biological system; IM wrote the first draft to which all authors contributed substantially.

\section{CONFLICT OF INTEREST}

The authors declare no conflict of interest.

\section{ACKNOWLEDGMENT}

This work was supported by H2020 European Research council (grant number 648143).

\section{DATA ARCHIVING}

The code supporting this study is archived at https://doi.org/10.5061/ dryad.0k6djhb0b.

\section{LITERATURE CITED}

Akbari, O. S., K. D. Matzen, J. M. Marshall, H. Huang, C. M. Ward, and B. A. Hay. 2013. A synthetic gene drive system for local, reversible modification and suppression of insect populations. Curr. Biol. 23:671677.

Brand, C. L., A. M. Larracuente, and D. C. Presgraves. 2015. Origin, evolution, and population genetics of the selfish Segregation Distorter gene duplication in European and African populations of Drosophila melanogaster. Evolution 69:1271-1283.

Buckler, E. S., T. L. Phelps-Durr, C. S. K. Buckler, R. K. Dawe, J. F. Doebley, and T. P. Holtsford. 1999. Meiotic drive of chromosomal knobs reshaped the maize genome. Genetics 153:415-426.

Bull, J. J. 2017. Lethal gene drive selects inbreeding. Evol. Med. Publ. Health 2017:1-16.

Bull, J. J., C. H. Remien, and S. M. Krone. 2019. Gene-drive-mediated extinction is thwarted by population structure and evolution of sib mating. Evol. Med. Publ. Health 2019:66-81.

Burt, A., and R. Trivers. 1998. Selfish DNA and breeding system in flowering plants. Proc. R. Soc. Lond. Ser. B 265:141-146.

.. 2006. Genes in conflict: the biology of selfish genetic elements. Harvard Univ. Press, Cambridge, MA.

Crow, J. F., and M. Kimura. 1970. An introduction to population genetics theory. Harper \& Row, New York.

Desai, M. M., and D. S. Fisher. 2007. Beneficial mutation-selection balance and the effect of linkage on positive selection. Genetics 176:1759-1798.

Didion, J. P., A. P. Morgan, A. M.-F. Clayshulte, R. C. Mcmullan, L. Yadgary, P. M. Petkov, T. A. Bell, D. M. Gatti, J. J. Crowley, K. Hua, et al. 2015. A multi-megabase copy number gain causes maternal transmission ratio distortion on mouse chromosome 2. PLoS Genet. 11:e1004850.

Esser, K. 1974. Podospora anserina. Pp. 531-551 in R. C. King, eds. Handbook of genetics. Vol. 1. Bacteria, bacteriophages, and fungi. Springer, New York.

Esvelt, K. M., A. L. Smidler, F. Catteruccia, and G. M. Church. 2014. Emerging technology: concerning RNA-guided gene drives for the alteration of wild populations. Elife 3:e03401.

Fishman, L., and J. K. Kelly. 2015. Centromere-associated meiotic drive and female fitness variation in Mimulus. Evolution 69:1208-1218.

Fishman, L., and A. Saunders. 2008. Centromere-associated female meiotic drive entails male fitness costs in monkeyflowers. Science 322:15591562 .

Frank, S. A. 1991. Divergence of meiotic drive-suppression systems as an explanation for sex-biased hybrid sterility and inviability. Evolution 45:262-267.

Gale, J. S. 1990. Theoretical population genetics. Unwin Hyman, London.

Grognet, P., H. Lalucque, F. Malagnac, and P. Silar. 2014. Genes that bias Mendelian segregation. PLoS Genet. 10:e1004387.

Hall, D. W., and R. K. Dawe. 2018. Modeling the evolution of female meiotic drive in maize. G3: Genes, Genomes, Genetics 8:123-130.

Hamilton, W. D. 1967. Extraordinary sex ratios. Science 156:477-488.

Hartl, D. L. 1970. Analysis of a general population genetic model of meiotic drive. Evolution 24:538-545.

.. 1972. Population dynamics of sperm and pollen killers. Theor. Appl. Genet. 42:81-88.

Henikoff, S., K. Ahmad, and H. S. Malik. 2001. The centromere paradox: stable inheritance with rapidly evolving DNA. Science 293:1098-1102.

Hiraizumi, Y. 1962. Distorted segregation and genetic load. Jpn. J. Genet. $37: 147-154$.

Holman, L., T. A. Price, N. Wedell, and H. Kokko. 2015. Coevolutionary dynamics of polyandry and sex-linked meiotic drive. Evolution 69:709720.

Holsinger, K. E., M. W. Feldman, and F. B. Christiansen. 1984. The evolution of self-fertilization in plants: a population genetic model. Am. Nat. 124:446-453.

Hu, W., Z.-D. Jiang, F. Suo, J.-X. Zheng, W.-Z. He, and L.-L. Du. 2017. A large gene family in fission yeast encodes spore killers that subvert Mendel's law. Elife 6:e26057.

Hurst, G. D. D., and J. H. Werren. 2001. The role of selfish genetic elements in eukaryotic evolution. Nat. Rev. Genet. 2:597-606. 
Kyrou, K., A. M. Hammond, R. Galizi, N. Kranjc, A. Burt, A. K. Beaghton, T. Nolan, and A. Crisanti. 2018. A CRISPR-Cas9 gene drive targeting doublesex causes complete population suppression in caged Anopheles gambiae mosquitoes. Nat. Biotechnol. 36:1062-1066.

Larracuente, A. M., and D. C. Presgraves. 2012. The selfish Segregation Distorter gene complex of Drosophila melanogaster. Genetics 192:33-53.

Lewontin, R. C. 1968. The effect of differential viability on the population dynamics of $t$ alleles in the house mouse. Evolution 22:262-273.

Lewontin, R. C., and L. C. Dunn. 1960. The evolutionary dynamics of a polymorphism in the house mouse. Genetics 45:705-722.

Lindholm, A. K., K. A. Dyer, R. C. Firman, L. Fishman, W. Forstmeier, L. Holman, H. Johannesson, U. Knief, H. Kokko, A. M. Larracuente, et al. 2016. The ecology and evolutionary dynamics of meiotic drive. Trends Ecol. Evol. 31:315-326.

Lyttle, T. W. 1991. Segregation distorters. Annu. Rev. Genet. 25:511-581.

_.. 1993. Cheaters sometimes prosper: distortion of mendelian segregation by meiotic drive. Trends Genet. 9:205-210.

Meade, L. C., D. Dinneen, R. Kad, D. M. Lynch, K. Fowler, and A. Pomiankowski. 2019. Ejaculate sperm number compensation in stalkeyed flies carrying a selfish meiotic drive element. Heredity 122:916926.

Nauta, M. J., and R. F. Hoekstra. 1993. Evolutionary dynamics of spore killers. Genetics 135:923-930.

Nieuwenhuis, B. P. S., and T. Y. James. 2016. The frequency of sex in fungi. Phil. Trans. R. Soc. B 371:20150540.

Nuckolls, N. L., M. A. B. Núñez, M. T. Eickbush, J. M. Young, J. J. Lange, S. Y. Jonathan, G. R. Smith, S. L. Jaspersen, H. S. Malik, and S. E. Zanders. 2017. wtf genes are prolific dual poison-antidote meiotic drivers. Elife 6:e26033.

Núñez, M. A. B., N. L. Nuckolls, and S. E. Zanders. 2018. Genetic villains: killer meiotic drivers. Trends Genet. 34:424-433.

Núñez, M. A. B., I. M. Sabbarini, M. T. Eickbush, Y. Liang, J. J. Lange, A. M. Kent, and S. E. Zanders. 2020. Dramatically diverse Schizosaccharomyces pombewtf meiotic drivers all display high gamete-killing efficiency. PLoS Genet. 16:e1008350.

Otto, S. P., and T. Day. 2011. A biologist's guide to mathematical modeling in ecology and evolution. Princeton Univ. Press, Princeton, NJ.

Padieu, E., and J. Bernet. 1967. Mode d'action des gènes responsables de l'avortement de certains produits de la méiose chez l'Ascomycète Podospora anserina. C. R. Acad. Hebd. Seances. Acad. Sci. D 264:23002303.

Pinzone, C. A., and K. A. Dyer. 2013. Association of polyandry and sex-ratio drive prevalence in natural populations of Drosophila neotestacea. Proc. R. Soc. B 280:20131397.

Raju, N. B. 1994. Ascomycete spore killers: chromosomal elements that distort genetic ratios among the products of meiosis. Mycologia 86:461473.

Raju, N. B., and D. D. Perkins. 1994. Diverse programs of ascus development in pseudohomothallic species of neurospora, gelasinospora, and podospora. Dev. Genet. 15:104-118.
Rice, W. R. 2013. Nothing in genetics makes sense except in light of genomic conflict. Annu. Rev. Ecol. Evol. Syst. 44:217-237.

Rizet, G., and C. Engelmann. 1949. Contribution à l'étude génétique d'un ascomycète tétrasporé: Podospora anserina. Rhem. Rv. Cytol. Biol. Veg. 11:201-304.

Sandler, L., and E. Novitski. 1957. Meiotic drive as an evolutionary force. Am. Nat. 91:105-110.

Silar, P. 2013. Podospora anserina: from laboratory to biotechnology. Pp. 283-309 in A. Benjamin, P. K. M. Horwitz, M. Mukherjee, P. Christian, and Kubicek, eds. Genomics of soil- and plant-associated fungi. Springer, Heidelberg.

Silver, L. M. 1985. Mouse $t$ haplotypes. Annu. Rev. Genet. 19:179-208.

Svedberg, J., A. A. Vogan, N. A. Rhoades, D. Sarmarajeewa, D. J. Jacobson, M. Lascoux, T. M. Hammond, and H. Johannesson. 2020. An introgressed gene causes meiotic drive in Neurospora sitophila. bioRxiv doi:10.1101/2020.01.29.923946.

Sweigart, A. L., Y. Brandvain, and L. Fishman. 2019. Making a murderer: the evolutionary framing of hybrid gamete-killers. Trend. Genet. 35:245252.

Turner, B. C., and D. D. Perkins. 1979. Spore killer, a chromosomal factor in Neurospora that kills meiotic products not containing it. Genetics 93:587-606.

Tusso, S., B. P. Nieuwenhuis, F. J. Sedlazeck, J. W. Davey, D. C. Jeffares, and J. B. Wolf. 2019. Ancestral admixture is the main determinant of global biodiversity in fission yeast. Mol. Biol. Evol. 36:1975-1989.

van der Gaag, M. 2005. Genomic conflicts in Podospora anserina= Genomische conflicten in Podospora anserina. Thesis, Wageningen University.

van der Gaag, M., A. J. M. Debets, J. Oosterhof, M. Slakhorst, J. A. G. M. Thijssen, and R. F. Hoekstra. 2000. Spore-killing meiotic drive factors in a natural population of the fungus Podospora anserina. Genetics 156:593-605.

Vogan, A. A., S. L. Ament-Velásquez, E. Bastiaans, O. Wallerman, S. J. Saupe, A. Suh, and H. Johannesson. 2020. The Enterprise: a massive transposon carrying Spok meiotic drive genes. bioRxiv doi:10.1101/2020.03.25.007153.

Vogan, A. A., S. L. Ament-Velásquez, A. Granger-Farbos, J. Svedberg, E. Bastiaans, A. J. Debets, V. Coustou, H. Yvanne, C. Clavé, S. J. Saupe, et al. 2019. Combinations of Spok genes create multiple meiotic drivers in Podospora. Elife 8:e46454.

Werren, J. H. 2011. Selfish genetic elements, genetic conflict, and evolutionary innovation. Proc. Natl. Acad. Sci. U.S.A. 108:10863-10870.

Zanders, S. E., M. T. Eickbush, S. Y. Jonathan, J.-W. Kang, K. R. Fowler, G. R. Smith, and H. S. Malik. 2014. Genome rearrangements and pervasive meiotic drive cause hybrid infertility in fission yeast. Elife 3: $\mathrm{e} 02630$.

Associate Editor: Prof. Michael Kopp

\section{Supporting Information}

Additional supporting information may be found online in the Supporting Information section at the end of the article.

Figure S1: Bifurcation analysis of the Neurospora model with haploid fitness costs $g$.

Figure S2: Invasion probability of a spore killer for the Neurospora model with haploid fitness costs $g$.

Figure S3: Bifurcation analysis of the Podospora model with additive $\left(h_{z}=0.5\right)$ diploid fitness costs $z$.

Figure S4: Bifurcation analysis of the Podospora model with dominant $\left(h_{z}=1\right)$ diploid fitness costs $z$. 
Figure S5: Bifurcation analysis of the Podospora model with recessive $\left(h_{k}=0\right)$ dikaryotic fitness costs $k$.

Figure S6: Bifurcation analysis of the Podospora model with additive $\left(h_{k}=0.5\right)$ dikaryotic fitness costs $k$.

Figure S7: Bifurcation analysis of the Podospora model with dominant $\left(h_{k}=1\right)$ dikaryotic fitness costs $k$.

Figure S8: Bifurcation analysis of the Podospora model with haploid fitness costs $g$.

Figure S9: Bifurcation analysis of the Podospora model with incomplete killing efficiency $(e=0.8)$ and recessive $\left(h_{z}=0\right)$ diploid fitness costs $z$. Figure S10: Bifurcation analysis of the Podospora model with incomplete killing efficiency $(e=0.8)$ and additive $\left(h_{z}=0.5\right)$ diploid fitness costs $z$. Figure S11: Bifurcation analysis of the Podospora model with monokaryons in 5\% of asci $(m=0.05)$ and recessive $\left(h_{z}=0\right)$ diploid fitness costs $z$. Figure S12: Bifurcation analysis of the Podospora model with monokaryons in 50\% of asci $(m=0.5)$ and recessive $\left(h_{z}=0\right)$ diploid fitness costs $z$. Figure S13: Invasion probability of a spore-killing allele Dfor the Podospora model with recessive $\left(h_{z}=0\right)$ diploid fitness costs $z$.

Figure S14: Invasion probability of a spore-killing allele Dfor the Podospora model with additive $\left(h_{z}=0.5\right)$ diploid fitness costs $z$.

Figure S15: Invasion probability of a spore-killing allele Dfor the Podospora model with dominant $\left(h_{z}=1\right)$ diploid fitness costs $z$.

Figure S16: Invasion probability of a spore-killing allele Dfor the Podospora model with recessive $\left(h_{k}=0\right)$ dikaryotic fitness costs $k$.

Figure S17: Invasion probability of a spore-killing allele Dfor the Podospora model with additive $\left(h_{k}=1 / 2\right)$ dikaryotic fitness costs $k$.

Figure S18: Invasion probability of a spore-killing allele Dfor the Podospora model with dominant $\left(h_{k}=1\right)$ dikaryotic fitness costs $k$.

Figure S19: Invasion probability of a spore-killing allele $D$ for the Podospora model with haploid fitness costs $g$.

Figure S20: Effect of population size on invasion probability of a spore-killing allele $D$ with recessive diploid costs $z$.

Figure S21: Frequency of Spok2, Spok3 and Spok4 in the Wageningen population of Podospora anserina.

Figure S22: Simplified life cycle of Podospora anserina and meiosis diagram. 